\title{
A genome-wide analysis of the small auxin- up RNA (SAUR) gene family in cotton
}

Xihua Li ${ }^{1,2}$, Guoyuan Liu', Yanhui Geng ${ }^{1}$, Man Wu' ${ }^{1}$, Wenfeng Pei ${ }^{1}$, Honghong Zhai ${ }^{1}$, Xinshan Zang ${ }^{1}$, Xingli Li ${ }^{1}$, Jinfa Zhang ${ }^{3^{*}}$, Shuxun $\mathrm{Yu}^{1,2^{*}}$ and Jiwen $\mathrm{Yu}^{1 *}$

\begin{abstract}
Background: Small auxin-up RNA (SAUR) gene family is the largest family of early auxin response genes in higher plants, which have been implicated in the regulation of multiple biological processes. However, no comprehensive analysis of SAUR genes has been reported in cotton (Gossypium spp.).

Results: In the study, we identified 145, 97, 214, and 176 SAUR homologous genes in the sequenced genomes of G. raimondii, G. arboreum, G. hirsutum, and G. barbadense, respectively. A phylogenetic analysis revealed that the SAUR genes can be classified into 10 groups. A further analysis of chromosomal locations and gene duplications showed that tandem duplication and segmental duplication events contributed to the expansion of the SAUR gene family in cotton. An exon-intron organization and motif analysis revealed the conservation of SAUR-specific domains, and the auxin responsive elements existed in most of the upstream sequences. The expression levels of 16 GhSAUR genes in response to an exogenous application of IAA were determined by a quantitative RT-PCR analysis. The genome-wide RNA-seq data and qRT-PCR analysis of selected SAUR genes in developing fibers revealed their differential expressions. The physical mapping showed that 20 SAUR genes were co-localized with fiber length quantitative trait locus (QTL) hotspots. Single nucleotide polymorphisms (SNPs) were detected for 12 of these 20 genes between G. hirsutum and G. barbadense, but no SNPs were identified between two backcross inbred lines with differing fiber lengths derived from a cross between the two cultivated tetraploids.
\end{abstract}

Conclusions: This study provides an important piece of genomic information for the SAUR genes in cotton and lays a solid foundation for elucidating the functions of SAUR genes in auxin signaling pathways to regulate cotton growth.

Keywords: Gossypium spp., Small auxin-up RNA (SAUR), Gene expression patterns, Fiber development

\section{Background}

Auxin, widely distributed in higher plants, influences nearly all aspects of plant growth and development through regulating cell division, expansion, differentiation, and patterning [1]. Studies in transcripts have revealed that early auxin responsive genes are induced by auxin within minutes [2]. Most of early regulated auxin responsive genes are classified into three families: Aux/IAA, Gretchen Hagen3 (GH3), and small auxin-up RNA (SAUR) [3]. Among these early auxin response genes,

\footnotetext{
*Correspondence: jinzhang@nmsu.edu; ysx195311@163.com; yujw666@hotmail.com

${ }^{3}$ Department of Plant and Environmental Sciences, New Mexico State University, Las Cruces 88003, USA

'State Key Laboratory of Cotton Biology, Institute of Cotton Research of CAAS, Anyang 455000, China

Full list of author information is available at the end of the article
}

SAURs are considered to be the most abundant. Apart from transcriptional regulations by auxin, many SAURs are also regulated post-transcriptionally as a conserved downstream destabilizing element (DST) in the 3'-untranslated region that confers high mRNA instability [4].

Since the first SAUR gene was identified in elongating soybean hypocotyl sections [5], members of this gene family have been identified by genome-wide analyses in diverse plant species, such as Arabidopsis [3], rice [6], sorghum [7], tomato [8], potato [8], maize [9], citrus [10], and ramie [11]. In total, more than 674 SAUR genes were identified from different species, but only a small portion of them have been functionally characterized. In Arabidopsis, the overexpression of AtSAUR19 subfamily proteins (AtSAUR19 to AtSAUR24) with a N-terminal tag resulted in root waving, increased hypocotyl elongation and leaf size, defective apical hook maintenance, and 
altered tropic responses [12]. A further analysis showed that AtSAUR19 stimulated plasma-membrane (PM) H ${ }^{+}$-ATPase activity to promote cell expansion by inhibiting the PP2C-D phosphatases [13, 14]. Other AtSAUR63 (AtSAUR61 to AtSAUR68 and AtSAUR75) subfamily proteins also led to long hypocotyls, petals and stamen filaments in transgenic Arabidopsis [15]. Gene AtSAUR36 has been reported to have a role in promoting leaf senescence [16]. In rice, the up-regulation of the OsSAUR39 gene negatively regulated auxin biosynthesis and transport [17]. Three other SAUR proteins, i.e., AtSAUR76, AtSAUR77 and AtSAUR78 affected ethylene receptor signaling and promoted plant growth and development upon auxin responses [18]. Recently, many light-induced and/or repressed SAUR genes were reported to mediate differential growth of cotyledons and hypocotyls [19]. These results have shown that SAUR proteins regulate diverse aspects of plant growth and development.

Cotton is one of the most important economic crops for its natural textile fiber in the world, as it produces the cotton fiber, a highly elongated cell derived from the ovule epidermis. Previous reports have shown that auxin plays an important role in fiber development [20, 21]. Specifically, the number of lint fibers was significantly increased with an overexpressed iaaM gene during fiber initiation in ovules [22]. However, no SAUR genes have been reported in cotton thus far. The genome sequences of the two tetraploid cotton species Gossypium hirsutum - AD1 [23, 24] and G. barbadense - AD2 [25, 26] and the two closest living extant relatives to their descendants G. raimondii - D5 $[27,28]$ and G. arboreum - A2 [29] provide an important genomic resource for a genome-wide analysis of gene families and other related genetic studies [30-32]. In this study, a genome-wide identification of SAUR genes in the four species with currently sequenced genomes was performed to characterize the SAUR gene family with respect to their structural, genomic and gene expression features. The results obtained in this study will provide new data for further studies on auxin signaling in cotton growth and development.

\section{Methods}

\section{Gene retrieval and characterization analysis}

The genome sequences of G. raimondii (JGI_v2.1), G. arboreum (BGI_v2.0), G. hirsutum acc. TM-1 (NAUNBI_v1.1) and G. barbadense acc. 3-79 (HAU-NBI_v1.0) were downloaded from the CottonGen website [33]. To identify potential SAUR proteins in the four cotton species, all the SAUR amino acid sequences from Arabidopsis, rice, sorghum, tomato, and potato, maize, citrus and ramie were used as query in local BLAST (with an E-value cut off of 1e-5) searches individually against the four cotton species genome databases. Next, candidate sequences were inspected with the HMMER software 3.0 with the HMM profiles of auxin-inducible signature domain structure (PF02519) and the pfam database [34] to confirm the presence of the conserved SAUR domain. Then, the ProtParam tool [35] was used to analyze the physicochemical parameters (i.e., length, molecular weight, and isoelectric point) of SAUR proteins [35]. Subcellular localization prediction was conducted using the CELLO v2.5 server [36].

\section{Phylogenetic analysis}

Multiple alignments for all of the available and predicted SAUR full-length protein sequences were performed using ClustalX2 with a manually adjustment where appropriate for the alignment of the SAUR domain. A phylogenetic tree was constructed using the Neighbor Joining (NJ) method of MEGA 6.0 [37] with the pairwise deletion option and a Poisson correction model. For a statistical reliability analysis, bootstrap tests were performed with 1000 replicates.

\section{Chromosomal locations and gene duplication analysis}

The physical chromosome locations of all SAUR genes were obtained from the genome sequence databases. Mapchart 2.2 [38] software was used to generate the chromosomal location image. The predicted SAUR proteins were first aligned by ClustalW2 at EMBL-EBI (http://www.ebi.ac.uk/Tools/msa/clustalw2/) prior to a gene duplication analysis. Gene duplication events were defined according to the following conditions: the alignment region covered more than $80 \%$ of the longer gene and the identity of the aligned regions was over $80 \%$ [30].

\section{Gene structure and conserved motif analysis}

The gene exon/intron structure was analyzed using the Gene Structure Display Server (GSDS) [39] by comparing the cDNAs with their corresponding genomic DNA sequences. The Multiple Em for Motif Elicitation (MEME; version 4.11.2) program was used to analyze the protein sequences of the four cotton species G. raimondii, G. arboreum, G. hirsutum, and G. barbadense designated GrSAURs, GaSAURs, GhSAURs, and GbSAURs, respectively. The following parameter settings were used: size distribution, zero or one occurrence per sequence; motifs count, 5 ; and motif width, between 6 and 37 wide.

\section{Search for upstream sequence elements}

For the promoter analysis, $2000 \mathrm{bp}$ of genomic DNA sequences upstream of the start codon (ATG) of each GhSAUR genes were downloaded from their genome sequence. The PLACE database [40] was used to search for cis-acting regulatory elements in the putative promoter regions. 


\section{Gene expression analysis}

The expression patterns of genes coding for GhSAURs in Upland cotton were analyzed in three developmental stages of two backcross inbred lines (BILs) (NMGA-062 and NMGA-105): 0 days post anthesis (DPA) ovules, 3 DPA ovules, and 10 DPA fibers, and two developmental stages of cultivar Xuzhou 142 and its fuzzless-lintless mutant Xuzhou $142 \mathrm{fl}:-3$ and 0 DPA ovules. The two BILs genotypes were derived from an interspecific backcrossing for two generations between Upland SG747 as the recurrent parent and G. barbadense Giza75 followed by selfing, and they had a significant difference in fiber length $[41,42]$. All the genotypes were grown in the Experimental Farm, Institute of Cotton Research (ICR), Chinese Academy of Agricultural Sciences (CAAS), Anyang, Henan province, China. Flowers at 0 DPA were tagged and harvested at $-3,0,3$ and 10 DPA to dissect ovules at $-3,0$, and $3 \mathrm{DPA}$ and developing fibers at 10 DPA. Three biological replications with 15 flowers per replication were used for each sampling stage. Ovules or fibers were immediately frozen in liquid nitrogen after dissection in the field and stored at $-80{ }^{\circ} \mathrm{C}$ until use. RNA from each tissue sample was extracted using an RNAprep Pure Plant kit per manufacturer's instructions (Tiangen, China). RNA quality and quantity were checked using an Agilent 2100 Bioanalyzer, then cDNA libraries were constructed. Fragments of 200-700 nt in size were paired-end sequenced using an Illumina HiSeq 2000 platform in BGI (Shenzhen, China) following the manufacturer's instructions. After removing adapter sequences and low-quality reads including these with more than $5 \%$ unknown nucleotides or with more than $20 \%$ nucleotides of sequencing quality $\leq 10$, the clean transcriptome sequencing data were submitted to the NCBI Sequence Read Archive (SRA) with the accession numbers SRP038911 and SRP039385 for NMGA-062 and NMGA-105, and accession number SRP056184 for Xuzhou 142 and Xuzhou 142 fl. Fragments per kilobase of transcript per million fragments (FPKM value) were calculated to normalize the expression level of each expressed GhSAUR gene based on gene length and the number of mapped reads. The formula is FPKM $=\frac{10^{6} \mathrm{C}}{N L / 10^{3}}$, where $\mathrm{C}$ is the number of fragments that are uniquely aligned to a specific gene, $\mathrm{N}$ is the total number of fragments that are uniquely aligned to all genes, and $\mathrm{L}$ is the number of bases on the specific gene. The expression profiles were clustered using the Cluster 3.0 software [43].

\section{Quantitative RT-PCR analysis}

To further characterize the expression of selected GhSAUR genes, tissue samples were collected at $-3,0,3$, $5,7,10,15,20$, and 25 DPA ovaries (i.e., bolls) from
NMGA-062 only and dissected for ovules at $-3,0$ and 3 DPA and fiber at other stages. The tissues were immediately frozen in liquid nitrogen and stored at $-80{ }^{\circ} \mathrm{C}$ until used for total RNA extraction. Root, stem, and leaf samples were also harvested. For each tissue sample, three biological replications were used.

To study the responses of selected GhSAURs to IAA application, seed from cultivar CCRI 10 (G. hirsutum) was planted in potting soil at $25{ }^{\circ} \mathrm{C}$ in a culture room with a 16-h light/8-h dark cycle. Young plants at the four-true leaf stage were treated with $100 \mathrm{mM}$ IAA, and control plants were sprayed with an equal volume of $\mathrm{ddH}_{2} \mathrm{O}$. Leaves were then harvested at $0,5,10$ and $30 \mathrm{~min}$, and $1 \mathrm{~h}$ after the treatment for RNA extraction. Three biological replications (10 plants per replication) were used for each time point.

Total RNA was isolated from various tissue samples with a Tiangen RNAprep Pure Plant kit (Tiangen, China) according to the manufacturer's instructions. The firststrand cDNA fragment was synthesized from total RNA using PrimeScript ${ }^{\oplus}$ RT Reagent kit (Takara, Japan). Then the cDNA templates were diluted 8 fold and used for quantitative RT-PCR (qRT-PCR). Gene specific primers (Additional file 1: Table S1) were designed using the Primer 5.0 software. The histone-3 gene (AF024716) was used as the internal control, as this gene as a reference gene has been commonly used in numerous studies in plants including cotton to verify different gene expression levels in various tissue samples $[44,45]$. The qRTPCR experiment with three replicates was performed on a Mastercycler ${ }^{\ominus}$ ep realplex (Eppendorf, German) in a volume of $20 \mu \mathrm{L}$ containing $10 \mu \mathrm{L}$ of $2 \times$ UltraSYBR Mixture (With ROX) (CWBIO, China), $6.2 \mu \mathrm{L}$ of RNaseFree water, $3 \mu \mathrm{L}$ of cDNA template, $400 \mathrm{nM}$ of forward and reverse primers into 96-well plates. The thermal cycling conditions were as follows: an initial denaturation step of $10 \mathrm{~min}$ at $95{ }^{\circ} \mathrm{C}$, followed by 40 cycles of $15 \mathrm{~s}$ at $95{ }^{\circ} \mathrm{C}$ for denaturation, $25 \mathrm{~s}$ at $60{ }^{\circ} \mathrm{C}$ for annealing and $30 \mathrm{~s}$ at $72{ }^{\circ} \mathrm{C}$ for extension. Then, the melting curve analysis was performed. The relative expression levels of genes were calculated using the $2^{-\Delta \Delta C T}$ method and normalized to the histone- 3 gene. The results were statistically analyzed using a t test.

\section{Co-localization of SAUR genes with quantitative trait loci (QTL) for fiber length (FL) and single nucleotide polymorphism (SNP) identification of FL-QTL co-localized SAUR genes}

To co-localize GhSAURs with QTL for FL, we downloaded molecular markers for FL QTL hotspots reported in interspecific $G$. hirsutum $\times$ G. barbadense populations [46] and markers for 4 FL QTLs reported in an BIL population containing the two BILs (NMGA-062 and NMGA-105) differing in fiber length [41]. Based on the 
anchoring marker's location in the G. hirsutum TM-1 genome [24], the positions of the corresponding QTL hotspots for FL in chromosomes and the 4 FL QTL regions identified in the BIL population were determined. Chromosome locations of SAUR genes within the FL QTL regions $(25 \mathrm{cM})$ were considered to be the targeted co-localized SAUR genes.

To identify SNPs, FL-QTL co-localized SAUR genes in allotetraploid G. hirsutum genome [24] were compared with homologous/allelic genes from two G. barbadense genomes [25, 26] G. raimondii genome [27], and G. arboreum genome [29] using a local Blastn program. Then, genes from the A2 genome and the At subgenome of $\mathrm{AD} 1$ and $\mathrm{AD} 2$ were aligned with ClustalX2 using default parameters, as with the genes from the D5 genome and the Dt subgenome of AD1 and AD2. SNPs between allotetraploids and diploids were identified manually for each QTL co-localized SAUR gene.

\section{Results}

Identification of SAUR gene family in two diploid and two allotetraploid cotton

With all the SAUR amino acid sequences from Arabidopsis (67), rice (56), sorghum (71), tomato (99), potato (134), maize (79), citrus (70) and ramie (71) as query, a Blast search against the CDS data identified a total of 157, 107, 227, and 192 predicted SAUR sequences in G. raimondii, G. arboreum, G. hirsutum, and G. barbadense, respectively. The putative SAUR members were then analyzed for conserved domains using the HMMER and Pfam programs, leading to the identification of 145 , 97, 214, and 176 SAUR genes in G. raimondii, G. arboreum, G. hirsutum, and G. barbadense, respectively (Table 1; Additional files 2, 3 and 4: Table S2, S3, S4). These genes were named consecutively from GrSAUR1 to GrSAUR145, GaSAUR1 to GaSAUR97, GhSAUR1 to GhSAUR214, and GbSAUR1 to GbSAUR176 for the four species, respectively, according to the order of their corresponding chromosomal locations. More than $97.5 \%$ of the 632 identified SAUR genes encode proteins ranging between 64 to 185 amino acids (AA), except for 16 genes with different lengths, i.e., less than 64 or more than 185 AA (i.e., GrSAUR140 encoding protein with 53 AA, GbSAUR31, GrSAUR109, GbSAUR27, GbSAUR158, GrSAUR40, GbSAUR91, GhSAUR126, GrSAUR134, GbSAUR161, GASAUR43, GbSAUR13, GrSAUR138, GbSAUR125, GhSAUR114 and GbSAUR34 encoding proteins with 190, 198, 199, 204, 206, 226, 228, 232, 232, 232, 239, 240, 242, 252, 295 and 376 AA, respectively). The predicted SAUR genes encode proteins with the predicted molecular weight $(\mathrm{Mw})$ ranging from 6.06 to $43.11 \mathrm{kDa}$ and the theoretical isoelectric point $(\mathrm{pI})$ varying between 4.69 and 11.42. The protein subcellular localization prediction showed that 397 of the 632 SAUR proteins were located in the nucleus, while others were plasma membrane, cytoplasmic, mitochondrial, chloroplast, or extracellular localized.

\section{Phylogenetic analysis of the SAUR gene family}

To study the phylogenetic relationship of the SAUR family, we performed a phylogenetic analysis of 647 SAUR protein sequences from Arabidopsis, rice, maize, tomato, potato, sorghum, citrus, and ramie and 632 cotton SAURs by generating a phylogenetic tree. As shown in Fig. 1, the SAUR proteins can be placed into 10 distinct groups, designated from group I to $\mathrm{X}$, which were based on their sequence similarities with orthologs in other plants. Group I and VII contained 365 and 237 SAUR members, respectively, and constituted the two largest groups in the phylogeny, while group III and V contained only 24 and 30 members, respectively. Each group contained SAURs from at least 9 plant species. Interestingly, group VI only contained SAURs from dicot species, i.e., G. raimondii, G. arboreum, G. hirsutum, G. barbadense, Arabidopsis, tomato, potato, citrus, and ramie as seen in Additional file 5: Table S5.

\section{Chromosomal distribution and duplication events of SAUR genes in cotton}

Using the genome sequences of the four cotton species as references, the identified 632 SAUR genes were mapped onto chromosomes or scaffolds. Of which, 543 of the 632 SAURs were assigned to chromosomes, while the remaining 89 SAURs were located in unmapped scaffolds (Fig. 2). Of the 133 GrSAURs mapped to 13 chromosomes of G. raimondii, chromosome D5_chr5 (with 31 genes) and chromosome D5_chr1 (with 21 genes) had the most number of SAUR genes, while D5_chr3 had only 1 SAUR gene. The 97 predicted SAUR genes in G. arboreum were also distributed unevenly across its 13 chromosomes, with chromosome A2_chr5 (similar to the homoeologous chromosome D5_chr5 in G. raimondii) harboring more genes (31) and chromosome A2_chr4 harboring the least genes (2). A total of 185 GhSAUR genes were mapped to 25 chromosomes of the G. hirsutum genome with 1 to 34 genes per chromosome, except for no SAUR genes identified on chromosome AD1_A02 in the At subgenome. Similarly, more SAUR genes were clustered on chromosomes AD1_A03, AD1_D02, AD1_D05, and AD1_D13. A total of 128 GbSAUR genes distributed unevenly over all the G. barbadense genome except for chromosome AD2_A02. The number of genes per chromosome ranged from 1 (chromosome AD2_A11) to 18 (chromosome AD2_D02).

To elucidate the expanded mechanism of the SAUR gene family, we performed a gene duplication event analysis including tandem duplication and segmental duplication in the four cotton species. After multiple and 
Table 1 The SAUR gene family in Gossypium raimondii

\begin{tabular}{|c|c|c|c|c|c|c|c|c|}
\hline Gene ID & Name & $\begin{array}{l}\text { Chromosome } \\
\text { number }\end{array}$ & Location & Intron & $\begin{array}{l}\text { Length } \\
\mathrm{AA}\end{array}$ & $\begin{array}{l}\mathrm{MW/} \\
\mathrm{kDa}\end{array}$ & $\mathrm{Pl}$ & Predicted subcellular localization \\
\hline GrSAUR1 & Gorai.001G017500.1 & Chr1 & $\begin{array}{l}1,615,768- \\
1,617,374(+)\end{array}$ & 1 & 157 & 17.92 & 5.97 & Nuclear(1.616)/Cytoplasmic(1.523) \\
\hline GrSAUR2 & Gorai.001G017600.1 & Chr1 & $\begin{array}{l}1,629,855- \\
1,630,662(-)\end{array}$ & 0 & 124 & 13.93 & 5.59 & Nuclear(2.037)/Mitochondrial(1.408) \\
\hline GrSAUR3 & Gorai.001G056100.1 & Chr1 & $\begin{array}{l}5,438,812- \\
5,439,758(+)\end{array}$ & 0 & 174 & 19.53 & 8.9 & Nuclear(3.349) \\
\hline GrSAUR4 & Gorai.001G244500.1 & Chr1 & $\begin{array}{l}48,767,330- \\
48,768,039(-)\end{array}$ & 0 & 104 & 11.89 & 8.91 & Mitochondrial(2.554) \\
\hline GrSAUR5 & Gorai.001G244700.1 & Chr1 & $\begin{array}{l}48,849,478- \\
48,849,772(-)\end{array}$ & 0 & 71 & 8.82 & 5.7 & Extracellular(1.110)/Cytoplasmic(1.063) \\
\hline GrSAUR6 & Gorai.001G245000.1 & Chr1 & $\begin{array}{l}48,885,401- \\
48,885,678(-)\end{array}$ & 1 & 80 & 9.17 & 10.46 & Mitochondrial(2.153) \\
\hline GrSAUR7 & Gorai.001G245100.1 & Chr1 & $\begin{array}{l}48,949,120- \\
48,949,404(-)\end{array}$ & 0 & 94 & 10.56 & 9.25 & Mitochondrial(1.764)/PlasmaMembrane(1.598) \\
\hline GrSAUR8 & Gorai.001G245200.1 & Chr1 & $\begin{array}{l}48,953,747- \\
48,953,998(+)\end{array}$ & 0 & 83 & 9.21 & 8.69 & Mitochondrial(2.051) \\
\hline GrSAUR9 & Gorai.001G245300.1 & Chr1 & $\begin{array}{l}48,959,152- \\
48,959,467(-)\end{array}$ & 0 & 95 & 10.70 & 8.71 & Mitochondrial(1.715)/Nuclear(1.086) \\
\hline GrSAUR10 & Gorai.001G245400.1 & Chr1 & $\begin{array}{l}49,022,365- \\
49,022,661(-)\end{array}$ & 1 & 86 & 9.67 & 8.98 & Mitochondrial(1.735)/Nuclear(1.232) \\
\hline GrSAUR11 & Gorai.001G245500.1 & Chr1 & $\begin{array}{l}49,029,187- \\
49,029,450(-)\end{array}$ & 0 & 87 & 9.87 & 10.29 & Mitochondrial(2.957) \\
\hline GrSAUR12 & Gorai.001G245600.1 & Chr1 & $\begin{array}{l}49,036,609- \\
49,036,207(-)\end{array}$ & 0 & 98 & 11.02 & 9.58 & Mitochondrial(2.247) \\
\hline GrSAUR13 & Gorai.001G245700.1 & Chr1 & $\begin{array}{l}49,037,471- \\
49,038,414(+)\end{array}$ & 0 & 79 & 8.97 & 6.71 & Nuclear(1.309)/Mitochondrial(1.055) \\
\hline GrSAUR14 & Gorai.001G245800.1 & Chr1 & $\begin{array}{l}49,039,305- \\
49,040,136(+)\end{array}$ & 0 & 96 & 10.67 & 7.82 & Chloroplast(1.497)/Mitochondrial(1.317) \\
\hline GrSAUR15 & Gorai.001G246200.1 & Chr1 & $\begin{array}{l}49,068,911- \\
49,069,132(-)\end{array}$ & 0 & 73 & 8.29 & 5.87 & Cytoplasmic(1.044) \\
\hline GrSAUR16 & Gorai.001G246300.1 & Chr1 & $\begin{array}{l}49,083,746- \\
49,084,276(-)\end{array}$ & 0 & 72 & 8.17 & 9.99 & Mitochondrial(2.350) \\
\hline GrSAUR17 & Gorai.001G246400.1 & Chr1 & $\begin{array}{l}49,089,129- \\
49,089,840(+)\end{array}$ & 0 & 86 & 9.37 & 9.65 & Mitochondrial(2.239) \\
\hline GrSAUR18 & Gorai.001G246500.1 & Chr1 & $\begin{array}{l}49,093,160- \\
49,094,276(+)\end{array}$ & 0 & 96 & 10.73 & 9.65 & Mitochondrial(2.601) \\
\hline GrSAUR19 & Gorai.001G246600.1 & Chr1 & $\begin{array}{l}49,103,722- \\
49,104,359(+)\end{array}$ & 0 & 101 & 11.52 & 9.56 & Mitochondrial(3.427) \\
\hline GrSAUR20 & Gorai.001G246700.1 & Chr1 & $\begin{array}{l}49,113,411- \\
49,114,457(+)\end{array}$ & 0 & 94 & 10.53 & 6.26 & Mitochondrial(1.852) \\
\hline GrSAUR21 & Gorai.001G246800.1 & Chr1 & $\begin{array}{l}49,115,279- \\
49,116,348(+)\end{array}$ & 0 & 94 & 10.46 & 6.39 & Cytoplasmic(1.765)/Mitochondrial(1.270) \\
\hline GrSAUR22 & Gorai.002G036000.1 & Chr2 & $280,592-2,807,117(+)$ & 0 & 144 & 17.05 & 8.41 & Nuclear(2.870) \\
\hline GrSAUR23 & Gorai.002G106000.1 & Chr2 & $\begin{array}{l}14,004,508- \\
14,005,911(+)\end{array}$ & 0 & 141 & 16.09 & 9.34 & Mitochondrial(1.938) \\
\hline GrSAUR24 & Gorai.002G124000.1 & Chr2 & $\begin{array}{l}18,059,123- \\
18,060,363(+)\end{array}$ & 0 & 106 & 12.06 & 7.81 & Mitochondrial(1.608)/Nuclear(1.500) \\
\hline GrSAUR25 & Gorai.002G124300.1 & Chr2 & $\begin{array}{l}18,174,772- \\
18,175,224(-)\end{array}$ & 0 & 150 & 17.25 & 9.71 & Mitochondrial(2.042) \\
\hline GrSAUR26 & Gorai.002G162000.1 & Chr2 & $\begin{array}{l}37,452,379- \\
37,453,133(+)\end{array}$ & 0 & 103 & 11.78 & 9.74 & Mitochondrial(2.171) \\
\hline GrSAUR27 & Gorai.002G202800.1 & Chr2 & $\begin{array}{l}54,437,591- \\
54,438,058(-)\end{array}$ & 0 & 155 & 17.46 & 8.98 & Mitochondrial(1.307)/Nuclear(1.228) \\
\hline
\end{tabular}


Table 1 The SAUR gene family in Gossypium raimondii (Continued)

\begin{tabular}{|c|c|c|c|c|c|c|c|c|}
\hline Gene ID & Name & $\begin{array}{l}\text { Chromosome } \\
\text { number }\end{array}$ & Location & Intron & $\begin{array}{l}\text { Length } \\
\text { AA }\end{array}$ & $\begin{array}{l}\mathrm{MW} / \\
\mathrm{kDa}\end{array}$ & $\mathrm{PI}$ & Predicted subcellular localization \\
\hline GrSAUR28 & Gorai.002G202900.1 & Chr2 & $\begin{array}{l}54,439,265- \\
54,439,708(-)\end{array}$ & 0 & 147 & 16.32 & 9.71 & Mitochondrial(1.306)/PlasmaMembrane(1.221) \\
\hline GrSAUR29 & Gorai.002G203000.1 & Chr2 & $\begin{array}{l}54,441,032- \\
54,441,484(-)\end{array}$ & 0 & 150 & 16.87 & 9.59 & $\begin{array}{l}\text { Mitochondrial(1.562)/PlasmaMembrane(1.028), } \\
\text { Nuclear(1.009) }\end{array}$ \\
\hline GrSAUR30 & Gorai.002G203100.1 & Chr2 & $\begin{array}{l}54,442,712- \\
54,443,320(-)\end{array}$ & 0 & 151 & 17.02 & 9.27 & Mitochondrial(1.582)/PlasmaMembrane(1.248) \\
\hline GrSAUR31 & Gorai.003G168900.1 & Chr3 & $\begin{array}{l}43,938,904- \\
43,939,884(-)\end{array}$ & 0 & 160 & 18.83 & 10.61 & Mitochondrial(2.278)/Nuclear(1.583) \\
\hline GrSAUR32 & Gorai.004G044200.1 & Chr4 & $\begin{array}{l}3,855,770- \\
3,856,615(+)\end{array}$ & 0 & 121 & 14.26 & 7.3 & Nuclear(2.002) \\
\hline GrSAUR33 & Gorai.004G123800.1 & Chr4 & $\begin{array}{l}32,179,516- \\
32,180,140(+)\end{array}$ & 1 & 132 & 15.66 & 6.85 & Mitochondrial(1.572)/Nuclear(1.337) \\
\hline GrSAUR34 & Gorai.004G149700.1 & Chr4 & $\begin{array}{l}42,322,441- \\
42,322,797(-)\end{array}$ & 0 & 118 & 14.00 & 9.01 & Nuclear(1.977)/Mitochondrial(1.434) \\
\hline GrSAUR35 & Gorai.004G163300.1 & Chr4 & $\begin{array}{l}45,433,271- \\
45,433,723(-)\end{array}$ & 1 & 132 & 15.54 & 9.79 & Mitochondrial(1.904) \\
\hline GrSAUR36 & Gorai.004G163400.1 & Chr4 & $\begin{array}{l}45,497,584- \\
45,498,096(-)\end{array}$ & 0 & 170 & 19.63 & 10.24 & Mitochondrial(2.920) \\
\hline GrSAUR37 & Gorai.004G201200.1 & Chr4 & $\begin{array}{l}52,760,164- \\
52,761,207(+)\end{array}$ & 0 & 163 & 18.99 & 10.17 & Mitochondrial(2.278) \\
\hline GrSAUR38 & Gorai.004G269500.1 & Chr4 & $\begin{array}{l}60,455,793- \\
60,456,903(-)\end{array}$ & 0 & 142 & 15.91 & 7.82 & Mitochondrial(1.415) \\
\hline GrSAUR39 & Gorai.004G285600.1 & Chr4 & $\begin{array}{l}61,641,514- \\
61,641,950(+)\end{array}$ & 1 & 101 & 11.42 & 8.95 & $\begin{array}{l}\text { Nuclear(1.681)/Mitochondrial(1.473)/ } \\
\text { Cytoplasmic(1.115) }\end{array}$ \\
\hline GrSAUR40 & Gorai.005G249200.1 & Chr5 & $\begin{array}{l}62,880,875- \\
62,882,624(+)\end{array}$ & 1 & 206 & 23.97 & 10.25 & Mitochondrial(1.433)/Nuclear(1.317) \\
\hline GrSAUR41 & Gorai.005G249400.1 & Chr5 & $\begin{array}{l}62,904,501- \\
62,905,022(-)\end{array}$ & 0 & 79 & 8.92 & 7.88 & Mitochondrial(1.395) \\
\hline GrSAUR42 & Gorai.005G249500.1 & Chr5 & $\begin{array}{l}62,906,555- \\
62,906,794(-)\end{array}$ & 0 & 79 & 8.90 & 8.93 & Mitochondrial(1.189)/Nuclear(1.011) \\
\hline GrSAUR43 & Gorai.005G249700.1 & Chr5 & $\begin{array}{l}62,909,228- \\
62,910,023(-)\end{array}$ & 0 & 79 & 8.95 & 7.89 & Mitochondrial(1.276)/Nuclear(1.242) \\
\hline GrSAUR44 & Gorai.005G249800.1 & Chr5 & $\begin{array}{l}62,911,828- \\
62,912,064(-)\end{array}$ & 0 & 78 & 8.85 & 7.89 & Mitochondrial(1.438)/Nuclear(1.297) \\
\hline GrSAUR45 & Gorai.005G249900.1 & Chr5 & $\begin{array}{l}62,916,783- \\
62,917,022(-)\end{array}$ & 0 & 79 & 8.91 & 6.71 & Mitochondrial(1.261)/Nuclear(1.177) \\
\hline GrSAUR46 & Gorai.005G250100.1 & Chr5 & $\begin{array}{l}62,926,267- \\
62,927,447(+)\end{array}$ & 0 & 79 & 8.94 & 9.21 & Mitochondrial(1.812) \\
\hline GrSAUR47 & Gorai.005G250200.1 & Chr5 & $\begin{array}{l}62,934,108- \\
62,934,684(+)\end{array}$ & 0 & 79 & 8.83 & 6.53 & $\begin{array}{l}\text { Extracellular(1.406)/Nuclear(1.177)/ } \\
\text { Mitochondrial(1.039) }\end{array}$ \\
\hline GrSAUR48 & Gorai.005G250300.1 & Chr5 & $\begin{array}{l}62,935,399- \\
62,936,252(+)\end{array}$ & 0 & 113 & 12.99 & 9.03 & PlasmaMembrane(3.361) \\
\hline GrSAUR49 & Gorai.005G250400.1 & Chr5 & $\begin{array}{l}62,937,212- \\
62,938,166(+)\end{array}$ & 0 & 78 & 8.81 & 7.89 & Nuclear(1.690)/Mitochondrial(1.319) \\
\hline GrSAUR50 & Gorai.005G250500.1 & Chr5 & $\begin{array}{l}62,939,729- \\
62,940,314(+)\end{array}$ & 0 & 79 & 8.91 & 7.89 & Mitochondrial(1.545)/Nuclear(1.283) \\
\hline GrSAUR51 & Gorai.005G250600.1 & Chr5 & $\begin{array}{l}62,941,478- \\
62,941,910(+)\end{array}$ & 0 & 79 & 8.81 & 6.71 & Mitochondrial(1.311)/Nuclear(1.032) \\
\hline GrSAUR52 & Gorai.005G250700.1 & Chr5 & $\begin{array}{l}62,949,815- \\
62,950,331(+)\end{array}$ & 0 & 79 & 8.92 & 8.93 & Mitochondrial(1.237) \\
\hline GrSAUR53 & Gorai.005G250800.1 & Chr5 & $\begin{array}{l}62,951,581- \\
62,952,293(-)\end{array}$ & 0 & 92 & 10.33 & 6.55 & $\begin{array}{l}\text { Mitochondrial(1.594)/PlasmaMembrane } \\
\text { (1.012) }\end{array}$ \\
\hline GrSAUR54 & Gorai.005G250900.1 & Chr5 & & 0 & 96 & 10.68 & 9.3 & Mitochondrial(2.249) \\
\hline
\end{tabular}


Table 1 The SAUR gene family in Gossypium raimondii (Continued)

\begin{tabular}{|c|c|c|c|c|c|c|c|c|}
\hline Gene ID & Name & $\begin{array}{l}\text { Chromosome } \\
\text { number }\end{array}$ & Location & Intron & $\begin{array}{l}\text { Length } \\
\text { AA }\end{array}$ & $\begin{array}{l}\mathrm{MW} / \\
\mathrm{kDa}\end{array}$ & $\mathrm{PI}$ & Predicted subcellular localization \\
\hline & & & $\begin{array}{l}62,960,626- \\
62,960,916(-)\end{array}$ & & & & & \\
\hline GrSAUR55 & Gorai.005G251000.1 & Chr5 & $\begin{array}{l}62,969,862- \\
62,969,303(+)\end{array}$ & 0 & 96 & 10.64 & 8.66 & $\begin{array}{l}\text { Mitochondrial(1.469)/Nuclear(1.215)/ } \\
\text { Extracellular(1.154) }\end{array}$ \\
\hline GrSAUR56 & Gorai.005G251100.1 & Chr5 & $\begin{array}{l}62,985,151- \\
62,985,441(+)\end{array}$ & 0 & 96 & 10.76 & 8.98 & Mitochondrial(2.622) \\
\hline GrSAUR57 & Gorai.005G251200.1 & Chr5 & $\begin{array}{l}62,989,841- \\
62,990,113(-)\end{array}$ & 0 & 90 & 10.32 & 7.81 & Nuclear(1.458) \\
\hline GrSAUR58 & Gorai.005G251400.1 & Chr5 & $\begin{array}{l}63,001,840- \\
63,002,157(-)\end{array}$ & 0 & 105 & 12.09 & 7.88 & Nuclear(1.720)/Mitochondrial(1.716) \\
\hline GrSAUR59 & Gorai.005G251500.1 & Chr5 & $\begin{array}{l}63,005,563- \\
63,006,419(+)\end{array}$ & 0 & 105 & 12.11 & 9.21 & Mitochondrial(2.949) \\
\hline GrSAUR60 & Gorai.005G251600.1 & Chr5 & $\begin{array}{l}63,014,837- \\
63,015,348(+)\end{array}$ & 0 & 94 & 10.57 & 7.76 & Mitochondrial(1.577)/Extracellular(1.068) \\
\hline GrSAUR61 & Gorai.005G251700.1 & Chr5 & $\begin{array}{l}63,016,684- \\
63,017,267(+)\end{array}$ & 0 & 104 & 11.72 & 9.03 & Mitochondrial(2.483) \\
\hline GrSAUR62 & Gorai.005G256400.1 & Chr5 & $\begin{array}{l}63,318,324- \\
63,318,851(+)\end{array}$ & 0 & 138 & 16.05 & 9.52 & Mitochondrial(1.593)/Nuclear(1.232) \\
\hline GrSAUR63 & Gorai.005G256500.1 & Chr5 & $\begin{array}{l}63,320,204- \\
63,320,849(+)\end{array}$ & 0 & 147 & 16.52 & 9.01 & Nuclear(1.268) \\
\hline GrSAUR64 & Gorai.005G256600.1 & Chr5 & $\begin{array}{l}63,345,303- \\
63,346,064(-)\end{array}$ & 0 & 147 & 16.61 & 9.42 & Nuclear(1.484) \\
\hline GrSAUR65 & Gorai.005G256700.1 & Chr5 & $\begin{array}{l}63,347,114- \\
63,348,038(-)\end{array}$ & 0 & 147 & 16.64 & 9.12 & Nuclear(1.348) \\
\hline GrSAUR66 & Gorai.005G256800.1 & Chr5 & $\begin{array}{l}63,349,316- \\
63,349,771(-)\end{array}$ & 0 & 151 & 17.25 & 9.28 & PlasmaMembrane(2.087) \\
\hline GrSAUR67 & Gorai.005G256900.1 & Chr5 & $\begin{array}{l}63,351,050- \\
63,351,454(-)\end{array}$ & 0 & 134 & 15.32 & 9.37 & $\begin{array}{l}\text { PlasmaMembrane(1.359)/Nuclear(1.193)/ } \\
\text { Mitochondrial(1.169) }\end{array}$ \\
\hline GrSAUR68 & Gorai.005G257000.1 & Chr5 & $\begin{array}{l}63,352,122- \\
63,352,535(-)\end{array}$ & 0 & 137 & 15.89 & 8.89 & Nuclear(1.742) \\
\hline GrSAUR69 & Gorai.005G257100.1 & Chr5 & $\begin{array}{l}63,354,207- \\
63,354,620(-)\end{array}$ & 0 & 137 & 15.99 & 9.52 & Mitochondrial(1.658)/Nuclear(1.285) \\
\hline GrSAUR70 & Gorai.005G257200.1 & Chr5 & $\begin{array}{l}63,356,605- \\
63,357,048(-)\end{array}$ & 0 & 147 & 16.47 & 9.32 & $\begin{array}{l}\text { Nuclear(1.401)/Chloroplast(1.342)/ } \\
\text { Mitochondrial(1.080) }\end{array}$ \\
\hline GrSAUR71 & Gorai.006G007200.1 & Chr6 & $\begin{array}{l}1,498,825- \\
1,499,363(+)\end{array}$ & 0 & 106 & 12.63 & 8.6 & Nuclear(2.192) \\
\hline GrSAUR73 & Gorai.006G154300.1 & Chr6 & $\begin{array}{l}41,306,397- \\
41,307,777(-)\end{array}$ & 2 & 127 & 14.51 & 9.21 & Nuclear(1.623)/Extracellular(1.064) \\
\hline GrSAUR72 & Gorai.006G154300.2 & Chr6 & $\begin{array}{l}41,306,397- \\
41,307,777(-)\end{array}$ & 0 & 127 & 14.42 & 5.91 & Cytoplasmic(1.635) \\
\hline GrSAUR74 & Gorai.006G163400.1 & Chr6 & $\begin{array}{l}42,370,463- \\
42,371,381(+)\end{array}$ & 0 & 133 & 15.08 & 5.07 & $\begin{array}{l}\text { Mitochondrial(1.441)/Nuclear(1.432)/ } \\
\text { Cytoplasmic(1.128) }\end{array}$ \\
\hline GrSAUR75 & Gorai.006G163500.1 & Chr6 & $\begin{array}{l}42,377,888- \\
42,378,232(+)\end{array}$ & 1 & 81 & 9.65 & 9.88 & $\begin{array}{l}\text { Mitochondrial(1.532)/Cytoplasmic(1.287)/ } \\
\text { Nuclear(1.193) }\end{array}$ \\
\hline GrSAUR76 & Gorai.006G163600.1 & Chr6 & $\begin{array}{l}42,404,212- \\
42,405,041(+)\end{array}$ & 0 & 133 & 15.05 & 5.07 & Mitochondrial(1.543)/Nuclear(1.368) \\
\hline GrSAUR77 & Gorai.006G163800.1 & Chr6 & $\begin{array}{l}42,412,170- \\
42,412,587(+)\end{array}$ & 1 & 126 & 14.30 & 8.49 & Nuclear(1.932)/Mitochondrial(1.702) \\
\hline GrSAUR78 & Gorai.006G163900.1 & Chr6 & $\begin{array}{l}42,419,577- \\
42,420,926(+)\end{array}$ & 0 & 112 & 12.88 & 4.85 & Cytoplasmic(1.491)/Nuclear(1.050) \\
\hline GrSAUR79 & Gorai.006G164000.1 & Chr6 & $\begin{array}{l}42,423,009- \\
42,423,594(+)\end{array}$ & 0 & 123 & 14.07 & 7.83 & Nuclear(2.049)/Mitochondrial(1.486) \\
\hline GrSAUR80 & Gorai.006G164100.1 & Chr6 & & 0 & 126 & 14.55 & 7.83 & Mitochondrial(1.905)/Nuclear(1.315) \\
\hline
\end{tabular}


Table 1 The SAUR gene family in Gossypium raimondii (Continued)

\begin{tabular}{|c|c|c|c|c|c|c|c|c|}
\hline Gene ID & Name & $\begin{array}{l}\text { Chromosome } \\
\text { number }\end{array}$ & Location & Intron & $\begin{array}{l}\text { Length } \\
\text { AA }\end{array}$ & $\begin{array}{l}\mathrm{MW} / \\
\mathrm{kDa}\end{array}$ & $\mathrm{PI}$ & Predicted subcellular localization \\
\hline & & & $\begin{array}{l}42,425,630- \\
42,426,271(+)\end{array}$ & & & & & \\
\hline GrSAUR81 & Gorai.006G165600.1 & Chr6 & $\begin{array}{l}42,539,210- \\
42,539,581(+)\end{array}$ & 0 & 123 & 14.15 & 9.08 & Extracellular(2.919) \\
\hline GrSAUR82 & Gorai.007G089900.1 & Chr7 & $\begin{array}{l}6,550,230- \\
6,550,733(+)\end{array}$ & 0 & 167 & 18.97 & 10.1 & Mitochondrial(2.525) \\
\hline GrSAUR83 & Gorai.007G195900.1 & Chr7 & $\begin{array}{l}19,409,462- \\
19,410,253(+)\end{array}$ & 0 & 105 & 12.00 & 8.62 & Mitochondrial(1.684)/Cytoplasmic(1.511) \\
\hline GrSAUR84 & Gorai.008G032500.1 & Chr8 & $\begin{array}{l}3,910,820- \\
3,911,852(+)\end{array}$ & 0 & 162 & 18.28 & 9.71 & Mitochondrial(1.971)/Nuclear(1.789) \\
\hline GrSAUR85 & Gorai.008G032600.1 & Chr8 & $\begin{array}{l}3,952,535- \\
3,953,523(-)\end{array}$ & 0 & 104 & 11.88 & 8.51 & Mitochondrial(2.231) \\
\hline GrSAUR86 & Gorai.008G113100.1 & Chr8 & $\begin{array}{l}34,515,914- \\
34,516,922(+)\end{array}$ & 0 & 164 & 18.72 & 10.64 & Mitochondrial(2.851) \\
\hline GrSAUR87 & Gorai.008G128100.1 & Chr8 & $\begin{array}{l}36,977,552- \\
36,978,549(+)\end{array}$ & 0 & 122 & 14.34 & 8.5 & Nuclear(2.376) \\
\hline GrSAUR88 & Gorai.008G157200.1 & Chr8 & $\begin{array}{l}41,984,144- \\
41,985,508(+)\end{array}$ & 0 & 154 & 17.45 & 9.34 & Nuclear(2.361) \\
\hline GrSAUR89 & Gorai.008G157200.2 & Chr8 & $\begin{array}{l}41,984,144- \\
41,985,508(+)\end{array}$ & 0 & 154 & 17.45 & 9.34 & Nuclear(2.361) \\
\hline GrSAUR90 & Gorai.008G208300.1 & Chr8 & $\begin{array}{l}49,321,783- \\
49,322,349(-)\end{array}$ & 0 & 139 & 15.64 & 9.32 & Nuclear(3.111) \\
\hline GrSAUR91 & Gorai.008G236200.1 & Chr8 & $\begin{array}{l}52,182,178- \\
52,183,143(-)\end{array}$ & 0 & 164 & 19.19 & 10.46 & Mitochondrial(2.156)/Nuclear(1.528) \\
\hline GrSAUR92 & Gorai.008G265900.1 & Chr8 & $\begin{array}{l}54,517,459- \\
54,518,713(-)\end{array}$ & 0 & 122 & 14.36 & 6.87 & Nuclear(2.160) \\
\hline GrSAUR93 & Gorai.009G037700.1 & Chr9 & $\begin{array}{l}2,776,385- \\
2,777,283(+)\end{array}$ & 0 & 163 & 18.43 & 7.73 & Nuclear(1.758)/Mitochondrial(1.092) \\
\hline GrSAUR94 & Gorai.009G037800.1 & Chr9 & $\begin{array}{l}2,778,553- \\
2,779,157(-)\end{array}$ & 0 & 123 & 13.74 & 5.35 & Nuclear(1.624)/Mitochondrial(1.303) \\
\hline GrSAUR95 & Gorai.009G144300.1 & Chr9 & $\begin{array}{l}10,942,378- \\
10,943,104(+)\end{array}$ & 0 & 140 & 15.57 & 5.02 & Nuclear(1.689)/Mitochondrial(1.031) \\
\hline GrSAUR96 & Gorai.009G189200.1 & Chr9 & $\begin{array}{l}14,553,243- \\
14,553,545(+)\end{array}$ & 0 & 100 & 11.45 & 10.38 & Mitochondrial(2.168) \\
\hline GrSAUR97 & Gorai.009G195800.1 & Chr9 & $\begin{array}{l}15,048,472- \\
15,050,245(+)\end{array}$ & 0 & 105 & 12.04 & 6.83 & Mitochondrial(1.586)/Nuclear(1.551) \\
\hline GrSAUR98 & Gorai.009G195800.2 & Chr9 & $\begin{array}{l}15,048,472- \\
15,050,245(+)\end{array}$ & 0 & 105 & 12.04 & 6.83 & Mitochondrial(1.586)/Nuclear(1.551) \\
\hline GrSAUR99 & Gorai.009G196000.1 & Chr9 & $\begin{array}{l}15,088,996- \\
15,089,460(-)\end{array}$ & 0 & 154 & 17.69 & 10.04 & Mitochondrial(2.329) \\
\hline GrSAUR100 & Gorai.009G270400.1 & Chr9 & $\begin{array}{l}22,574,039- \\
22,575,114(+)\end{array}$ & 0 & 104 & 11.90 & 9.06 & Mitochondrial(2.106)/Nuclear(1.457) \\
\hline GrSAUR101 & Gorai.009G270500.1 & Chr9 & $\begin{array}{l}22,595,674- \\
22,596,117(-)\end{array}$ & 0 & 147 & 17.02 & 9.57 & Mitochondrial(1.673)/Nuclear(1.267) \\
\hline GrSAUR102 & Gorai.009G289300.1 & Chr9 & $\begin{array}{l}24,863,485- \\
24,863,904(+)\end{array}$ & 0 & 82 & 9.59 & 9.3 & Nuclear(1.735)/Mitochondrial(1.401) \\
\hline GrSAUR103 & Gorai.009G352900.1 & Chr9 & $\begin{array}{l}45,477,526- \\
45,477,954(-)\end{array}$ & 0 & 142 & 16.29 & 9.12 & Mitochondrial(1.798)/Nuclear(1.133) \\
\hline GrSAUR104 & Gorai.009G400900.1 & Chr9 & $\begin{array}{l}57,808,384- \\
57,808,761(-)\end{array}$ & 0 & 125 & 13.88 & 6.73 & $\begin{array}{l}\text { Nuclear(1.308)/Cytoplasmic(1.148)/ } \\
\text { Mitochondrial(1.145) }\end{array}$ \\
\hline GrSAUR105 & Gorai.009G409300.1 & Chr9 & $\begin{array}{l}61,459,699- \\
61,458,422(+)\end{array}$ & 0 & 114 & 12.98 & 6.89 & Chloroplast(1.175)/Mitochondrial(1.173) \\
\hline GrSAUR106 & Gorai.009G416100.1 & Chr9 & & 0 & 117 & 13.55 & 8.46 & Extracellular(2.548) \\
\hline
\end{tabular}


Table 1 The SAUR gene family in Gossypium raimondii (Continued)

\begin{tabular}{|c|c|c|c|c|c|c|c|c|}
\hline Gene ID & Name & $\begin{array}{l}\text { Chromosome } \\
\text { number }\end{array}$ & Location & Intron & $\begin{array}{l}\text { Length } \\
\text { AA }\end{array}$ & $\begin{array}{l}\mathrm{MW} / \\
\mathrm{kDa}\end{array}$ & $\mathrm{Pl}$ & Predicted subcellular localization \\
\hline & & & $\begin{array}{l}64,103,565- \\
64,103,918(+)\end{array}$ & & & & & \\
\hline GrSAUR107 & Gorai.010G005300.1 & Chr10 & $238,684-239,735(+)$ & 0 & 110 & 12.62 & 8.62 & Nuclear(2.021)/Mitochondrial(1.406) \\
\hline GrSAUR108 & Gorai.010G005500.1 & Chr10 & $275,408-275,866(-)$ & 0 & 152 & 17.10 & 9.86 & Mitochondrial(2.642) \\
\hline GrSAUR109 & Gorai.010G091700.1 & Chr10 & $\begin{array}{l}14,495,966- \\
14,498,408(+)\end{array}$ & 3 & 198 & 22.32 & 5.29 & Nuclear(1.433)/Cytoplasmic(1.237) \\
\hline GrSAUR110 & Gorai.010G091700.2 & Chr10 & $\begin{array}{l}14,496,288- \\
14,498,454(+)\end{array}$ & 1 & 161 & 18.16 & 5.16 & Nuclear(1.969)/Cytoplasmic(1.322) \\
\hline GrSAUR114 & Gorai.010G091700.3 & Chr10 & $\begin{array}{l}14,496,388- \\
14,498,454(+)\end{array}$ & 1 & 161 & 18.16 & 5.16 & Nuclear(1.969)/Cytoplasmic(1.322) \\
\hline GrSAUR111 & Gorai.010G091700.4 & Chr10 & $\begin{array}{l}14,496,345- \\
14,498,408(+)\end{array}$ & 1 & 161 & 18.16 & 5.16 & Nuclear(1.969)/Cytoplasmic(1.322) \\
\hline GrSAUR113 & Gorai.010G091700.5 & Chr10 & $\begin{array}{l}14,496,367- \\
14,498,408(+)\end{array}$ & 1 & 161 & 18.16 & 5.16 & Nuclear(1.969)/Cytoplasmic(1.322) \\
\hline GrSAUR112 & Gorai.010G091700.6 & Chr10 & $\begin{array}{l}14,496,367- \\
14,498,408(+)\end{array}$ & 1 & 161 & 18.16 & 5.16 & Nuclear(1.969)/Cytoplasmic(1.322) \\
\hline GrSAUR115 & Gorai.010G091700.7 & Chr10 & $\begin{array}{l}14,496,598- \\
14,498,408(+)\end{array}$ & 1 & 161 & 18.16 & 5.16 & Nuclear(1.969)/Cytoplasmic(1.322) \\
\hline GrSAUR116 & Gorai.010G091700.8 & Chr10 & $\begin{array}{l}14,496,730- \\
14,498,408(+)\end{array}$ & 1 & 161 & 18.16 & 5.16 & Nuclear(1.969)/Cytoplasmic(1.322) \\
\hline GrSAUR117 & Gorai.010G091700.9 & Chr10 & $\begin{array}{l}14,497,040- \\
14,498,408(+)\end{array}$ & 1 & 161 & 18.16 & 5.16 & Nuclear(1.969)/Cytoplasmic(1.322) \\
\hline GrSAUR118 & Gorai.010G092200.1 & Chr10 & $\begin{array}{l}14,625,769- \\
14,626,573(-)\end{array}$ & 0 & 126 & 14.15 & 5.32 & Mitochondrial(1.386)/Nuclear(1.328) \\
\hline GrSAUR119 & Gorai.010G141400.1 & Chr10 & $\begin{array}{l}34,863,658- \\
34,864,380(+)\end{array}$ & 0 & 159 & 18.02 & 9.32 & Nuclear(2.768) \\
\hline GrSAUR120 & Gorai.010G170300.1 & Chr10 & $\begin{array}{l}49,488,730- \\
49,489,183(+)\end{array}$ & 0 & 95 & 11.19 & 8.66 & Mitochondrial(1.707)/Nuclear(1.373) \\
\hline GrSAUR121 & Gorai.010G235800.1 & Chr10 & $\begin{array}{l}60,631,341- \\
60,631,637(+)\end{array}$ & 0 & 98 & 11.76 & 9.73 & Cytoplasmic(2.066)/Mitochondrial(1.394) \\
\hline GrSAUR122 & Gorai.011G002200.1 & Chr11 & 193,299-193,996(-) & 0 & 176 & 19.75 & 9.01 & Nuclear(2.687) \\
\hline GrSAUR123 & Gorai.011G052800.1 & Chr11 & $\begin{array}{l}4,142,829- \\
4,143,791(+)\end{array}$ & 0 & 119 & 13.88 & 10.07 & Nuclear(2.189)/Mitochondrial(1.517) \\
\hline GrSAUR124 & Gorai.011G052800.2 & Chr11 & $\begin{array}{l}4,142,866- \\
4,143,738(+)\end{array}$ & 0 & 119 & 13.88 & 10.07 & Nuclear(2.189)/Mitochondrial(1.517) \\
\hline GrSAUR125 & Gorai.011G056900.1 & Chr11 & $\begin{array}{l}4,500,795- \\
4,501,702(+)\end{array}$ & 0 & 119 & 13.43 & 7.73 & $\begin{array}{l}\text { Mitochondrial(1.538)/Nuclear(1.274)/ } \\
\text { Chloroplast(1.092) }\end{array}$ \\
\hline GrSAUR126 & Gorai.011G099000.1 & Chr11 & $\begin{array}{l}10,972,095- \\
10,973,058(+)\end{array}$ & 0 & 104 & 11.83 & 9.23 & Cytoplasmic(1.774)/Mitochondrial(1.435) \\
\hline GrSAUR127 & Gorai.012G037000.1 & Chr12 & $\begin{array}{l}4,571,265- \\
4,571,576(-)\end{array}$ & 0 & 103 & 12.24 & 6.62 & Nuclear(1.767)/Cytoplasmic(1.069) \\
\hline GrSAUR128 & Gorai.012G153700.1 & Chr12 & $\begin{array}{l}32,746,689- \\
32,747,015(+)\end{array}$ & 0 & 108 & 12.54 & 9.43 & Mitochondrial(2.120) \\
\hline GrSAUR129 & Gorai.012G153800.1 & Chr12 & $\begin{array}{l}32,752,110- \\
32,752,454(+)\end{array}$ & 0 & 114 & 13.39 & 9.57 & Mitochondrial(2.160) \\
\hline GrSAUR130 & Gorai.013G141300.1 & Chr13 & $\begin{array}{l}38,291,563- \\
38,293,037(+)\end{array}$ & 0 & 143 & 16.31 & 8.9 & Nuclear(1.590)/Mitochondrial(1.264) \\
\hline GrSAUR131 & Gorai.013G141300.2 & Chr13 & $\begin{array}{l}38,291,616- \\
38,293,037(+)\end{array}$ & 0 & 143 & 16.31 & 8.9 & Nuclear(1.590)/Mitochondrial(1.264) \\
\hline GrSAUR132 & Gorai.013G222500.1 & Chr13 & $\begin{array}{l}54,241,671- \\
54,242,678(+)\end{array}$ & 0 & 124 & 13.89 & 4.78 & $\begin{array}{l}\text { Nuclear(1.349)/Mitochondrial(1.105)/ } \\
\text { Chloroplast(1.084) }\end{array}$ \\
\hline GrSAUR133 & Gorai.013G268800.1 & Chr13 & & 0 & 140 & 16.09 & 6.59 & Cytoplasmic(1.835)/Nuclear(1.509) \\
\hline
\end{tabular}


Table 1 The SAUR gene family in Gossypium raimondii (Continued)

\begin{tabular}{|c|c|c|c|c|c|c|c|c|}
\hline Gene ID & Name & $\begin{array}{l}\text { Chromosome } \\
\text { number }\end{array}$ & Location & Intron & $\begin{array}{l}\text { Length } \\
\text { AA }\end{array}$ & $\begin{array}{l}\mathrm{MW} / \\
\mathrm{kDa}\end{array}$ & $\mathrm{PI}$ & Predicted subcellular localization \\
\hline & & & $\begin{array}{l}58,076,512- \\
58,076,950(-)\end{array}$ & & & & & \\
\hline GrSAUR134 & Gorai.N011800.1 & scaffold_36 & $3329-4346(+)$ & 0 & 232 & 26.12 & 9.26 & PlasmaMembrane(2.573) \\
\hline GrSAUR135 & Gorai.N011900.1 & scaffold_36 & $5114-5805(-)$ & 0 & 140 & 16.18 & 9.57 & $\begin{array}{l}\text { Mitochondrial(1.418)/PlasmaMembrane(1.023)/ } \\
\text { Nuclear(1.022) }\end{array}$ \\
\hline GrSAUR136 & Gorai.N012000.1 & scaffold_36 & 7594-8037(-) & 0 & 147 & 16.52 & 9.06 & PlasmaMembrane(1.131)/Nuclear(1.029) \\
\hline GrSAUR137 & Gorai.N012100.1 & scaffold_36 & 9050-9965(-) & 0 & 147 & 16.73 & 9.19 & Nuclear(2.010) \\
\hline GrSAUR138 & Gorai.N012200.1 & scaffold_36 & $13,475-13,932(-)$ & 1 & 240 & 27.46 & 8.63 & PlasmaMembrane(3.312) \\
\hline GrSAUR139 & Gorai.N012300.1 & scaffold_36 & $11,601-14,876(-)$ & 0 & 99 & 11.53 & 10 & Mitochondrial(1.666)/PlasmaMembrane(1.257) \\
\hline GrSAUR140 & Gorai.N014500.1 & scaffold_108 & $3-164(+)$ & 0 & 53 & 6.06 & 9.69 & Mitochondrial(1.772) \\
\hline GrSAUR141 & Gorai.N014600.1 & scaffold_108 & $1558-2077(+)$ & 0 & 79 & 8.94 & 9.3 & Mitochondrial(1.680) \\
\hline GrSAUR142 & Gorai.N014700.1 & scaffold_108 & 2935-3846(+) & 0 & 81 & 9.15 & 6.55 & Nuclear(1.375)/Mitochondrial(1.293) \\
\hline GrSAUR143 & Gorai.N014800.1 & scaffold_108 & $5215-12,132(+)$ & 1 & 79 & 8.91 & 7.89 & Mitochondrial(1.707)/Nuclear(1.032) \\
\hline GrSAUR144 & Gorai.N014900.1 & scaffold_108 & 6574-7195(+) & 0 & 79 & 8.90 & 8.89 & Mitochondrial(1.519) \\
\hline GrSAUR145 & Gorai.N015000.1 & scaffold_108 & 9410-9987(+) & 0 & 78 & 8.81 & 6.71 & $\begin{array}{l}\text { Nuclear(1.433)/ } \\
\text { Extracellular(1.053)/Mitochondrial(1.017) }\end{array}$ \\
\hline
\end{tabular}

pairwise alignments of GrSAURs, GaASURs, GhSAURs and GbSAURs, we chose the paralogous genes with the criteria described in previous studies [30]. As a result, 98, 54, 187 and 144 putative paralogous SAUR genes with high gene identity and similarity were found, accounting for $67.6 \%, 55.7 \%, 87.4 \%$ and $81.8 \%$ of the entire SAUR gene family in G. raimondii, G. arboreum, G. hirsutum, and G. barbadense, respectively. We observed that tandem duplication and segmental duplication events contributed to the expansion of the SAUR gene family in cotton. The details for the duplicated gene pairs were listed in Additional file 6: Table S6. In G. raimondii, 14 pairs of tandem duplication and 21 pairs of segmental duplication events were detected. The clusters of tandem duplication were mainly on chromosome D2_chr5. In G. arboreum, 3 clusters of SAUR genes were produced by tandem duplications, and the clusters of genes were distributed on the same chromosome (i.e., A2_chr5). In the same species, 17 pairs of SAUR genes were produced by segmental duplications and the two genes in each pair were from different chromosomes. In G. hirsutum, 9 clusters of genes with tandem duplications were detected mainly on chromosome AD1_A03 and AD1_D02, and 75 pairs of genes with segmental duplication events were detected with the two genes from each pair distributed on different chromosomes. The most majority of GbSAUR genes in G. barbadense were found to occur from tandem duplication or segmental duplication events. There are 23 pairs of tandem duplication clusters existed mainly on chromosome AD2_A05 and AD2_D02. 23 gene pairs with segmental duplication all distributed on different chromosomes.
Interestingly, most of the tandem duplication events in GhSAURs occurred on chromosome AD1_D02. It is apparent that homeologous chromosome 5 in G. raimondii (D5) and G. arboreum (A2), and chromosome D02 in G. hirsutum (AD1) and G. barbadense (AD2) all harbored more SAUR genes derived from tandem duplications. Chromosome D02 from AD1 and AD2 may be also homeologous to A2_chr5. The tandem duplication gene pairs were shown in Fig. 3 except for genes that were not localized to a specific chromosome.

\section{Gene structure and conserved motifs}

To gain an insight into the diversification of the SAUR genes in cotton, the exon/intron organization and conserved motifs were further analyzed. Based on the evolutionary relationships (Fig. 4a, Additional files 7, 8 and 9: Figure S1A, S2A, S3A), the detailed structure features of SAUR genes were shown in Fig. $4 \mathrm{~b}$ and Additional files 7, 8 and 9: Figure S1B, S2B, S3B. In general, more than $90.7 \%$ of SAUR genes lacked introns, 19 genes in GrSAURs, 4 genes in GaSAURs, 11 genes in GhSAURs, and 19 genes in GbSAURs each had 1 intron. Only 4 genes, namely GrSAUR73, GaSAUR76, GhSAUR114, and GbSAUR34 each had 2 introns and another 2 genes (GrSAUR109 and GbSAUR13) each had 3 introns. Conserved motifs in the 632 SAUR proteins were identified using the MEME online tool (Fig. 4c, Additional files 7, 8 and 9: Figure S1C, S2C, S3C). Motifs 1, 2, and 3 constitute the conserved SAUR-specific domain of approximately 60 residues in the central region of the sequences and were identified in most of the predicted SAUR proteins. Motifs 4 and 5 accounted for $39.7 \%$ and 


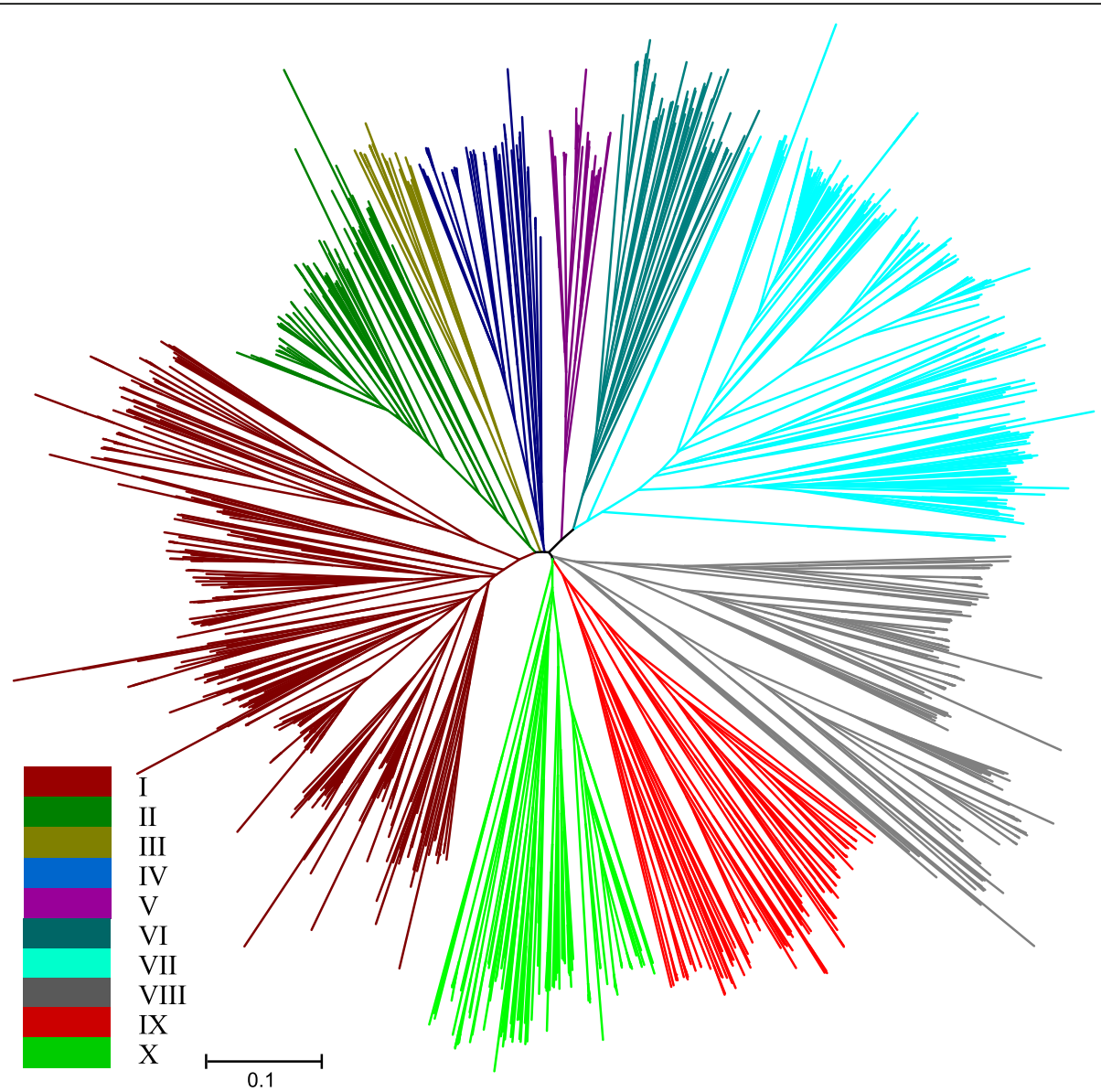

Fig. 1 A Phylogenetic tree of SAUR proteins from Gossypium raimondii, G. arboreum, G. hirsutum, G. barbadense, Arabidopsis, rice, maize, tomato, potato, sorghum, citrus, and ramie. The phylogenetic tree was generated using MEGA 6.0 with the Neighbour-Joining (NJ) method with 1000 bootstrap replicates. Different colored line marks groups I - X of the SAURs

$25.8 \%$ of the SAUR members, respectively, suggesting that these features might have contributed to some specific functions in the SAUR family.

Similar to the histidine-rich (H-rich) regions found in Arabidopsis, sorghum, tomato, and potato [3, 7, 8], the $\mathrm{H}$-rich regions were identified in the sequences of 39 predicted cotton SAUR genes. They are 7 GrSAURs, 6 GaSAURs, 14 GhSAURs, and 12 GbSAURs. The multiple alignments among these 39 SAUR proteins were shown in Additional file 10: Figure S4, and the H-rich regions were located on both or either of the $\mathrm{N}$-terminal and $\mathrm{C}$ terminal sequences.

\section{Promoter regions of GhSAUR genes}

The scanning of cis-acting regulatory DNA elements within promoter regions (2.0 kb from the start codon) of 165 randomly chosen GhSAUR genes was performed using the PLACE database. The results revealed that the promoters of the SAUR gene family contain numerous DNA elements predicted to be auxin signaling transduction related cis- elements. At least one of the seven major auxin-responsive cis-elements - S000024, S000026, S000234, S000270, S000273, S000360, and S000370, has been found in the promoter regions of the SAURs, except for 12 predicted GhSAURs genes. Another two regulatory sequences, i.e., $\mathrm{Ca}^{2+}$-responsive cis-element (S000501) and calmodulinbinding/CGCG box (S000507) were found in 47 predicted GhSAURs genes (Additional file 11: Table S7).

\section{Responses of SAUR genes in leaves to an exogenous IAA application}

The expression of SAUR genes is regulated at multiple levels in other reported species [3]. We analyzed the expression of 16 GhSAUR genes in leaves under exogenous IAA treatment (Fig. 5). 11 of these genes were upregulated at $5 \mathrm{~min}$ to $1 \mathrm{~h}$ after the IAA treatment, while 3 genes (GhSAUR56, GhSAUR61, and GhSAUR163) were down-regulated by the exogenous IAA application. Another 2 genes (GhSAUR63 and GhSAUR181) showed a relatively stable expression regardless of IAA treatment. 


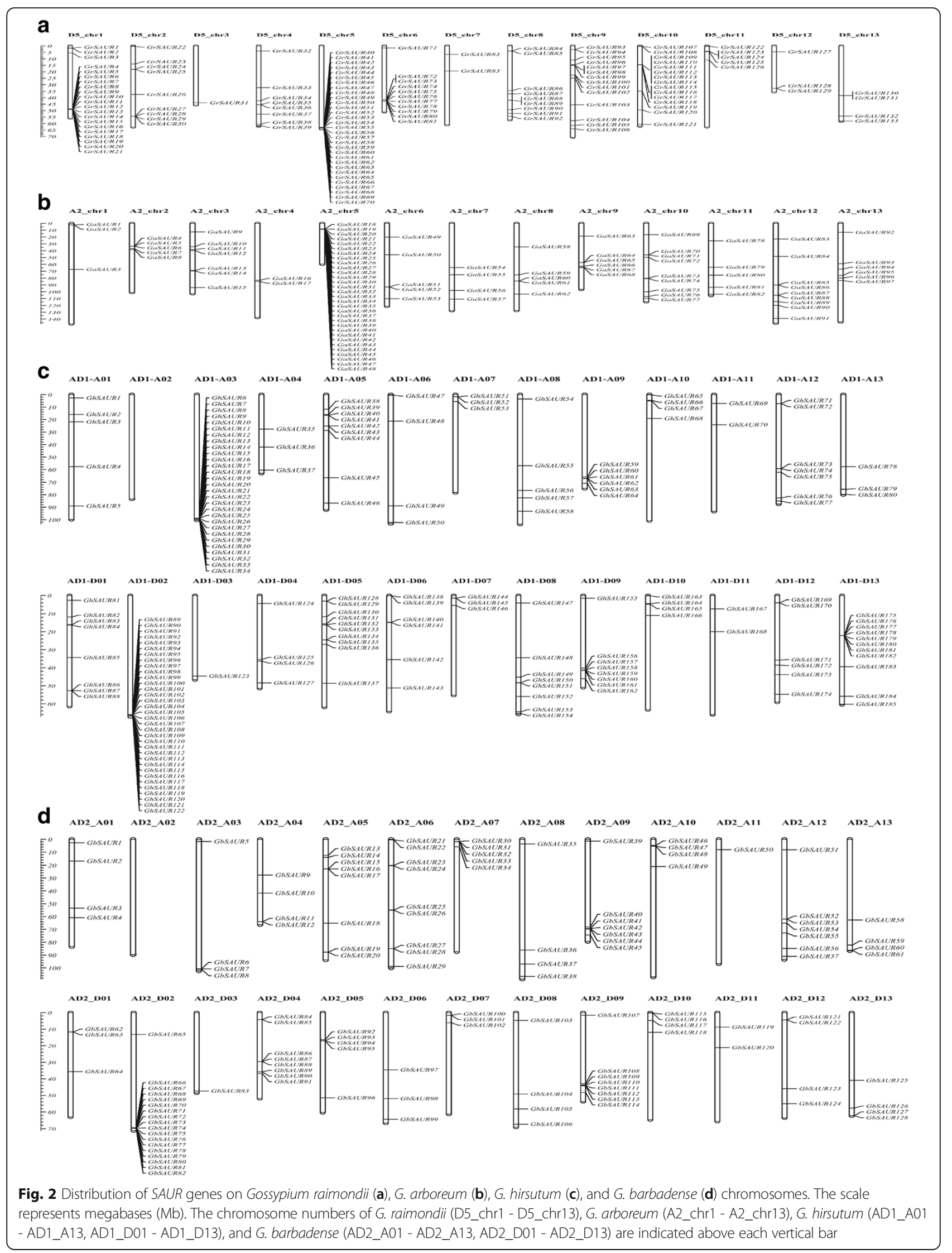




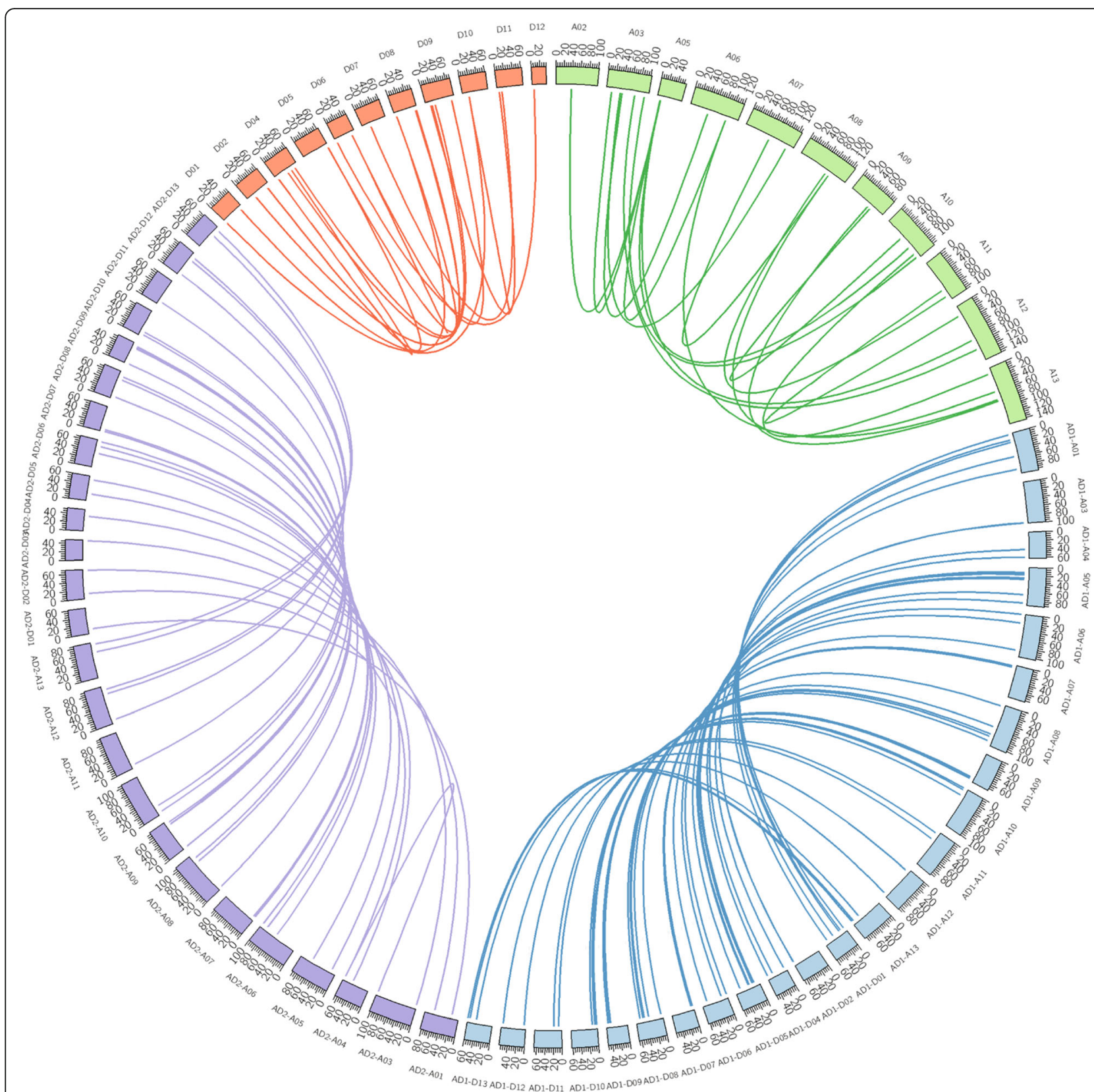

Fig. 3 The Circos diagram of paralogous gene pairs identified in GrSAURs, GaSAURs, GhSAURs, and GbSAURs. The chromosomes of Gossypium raimondii, G. arboreum, G. hirsutum, and G. barbadense were filled with red, green, blue, and purple colors, respectively. A line between two genes indicates a paralog

Therefore, the response of SAUR genes to IAA treatment in leaves varies, depending on SAUR genes.

\section{Expression characterization of GhSAUR genes in developing ovules and fibers}

The RNA-seq transcriptome data from the two backcross inbred lines (BILs) NMGA-062 and NMGA-105 at different developmental stages (0 DPA and 3 DPA ovules, and 10 DPA fibers) and Xuzhou142 and Xuzhou142 $f l$ mutant at -3 and 0 DPA ovules were used to analyze the expression patterns of candidate GhSAUR genes in Upland cotton. The NMGA-062 had a greater fiber length than NMGA-105. Among the 214 GhSAURs, 72 genes had an FPKM $\geq 1$ in at least one of the three developmental stages of the two BILs and were used to analyze the relative expression of each gene. Based on a cluster analysis, these SAUR genes showed four major patterns (Fig. 6a). The first group is composed of 20 genes showing an overall higher level in 0 DPA and 3 DPA ovules and 10 DPA fibers, when 


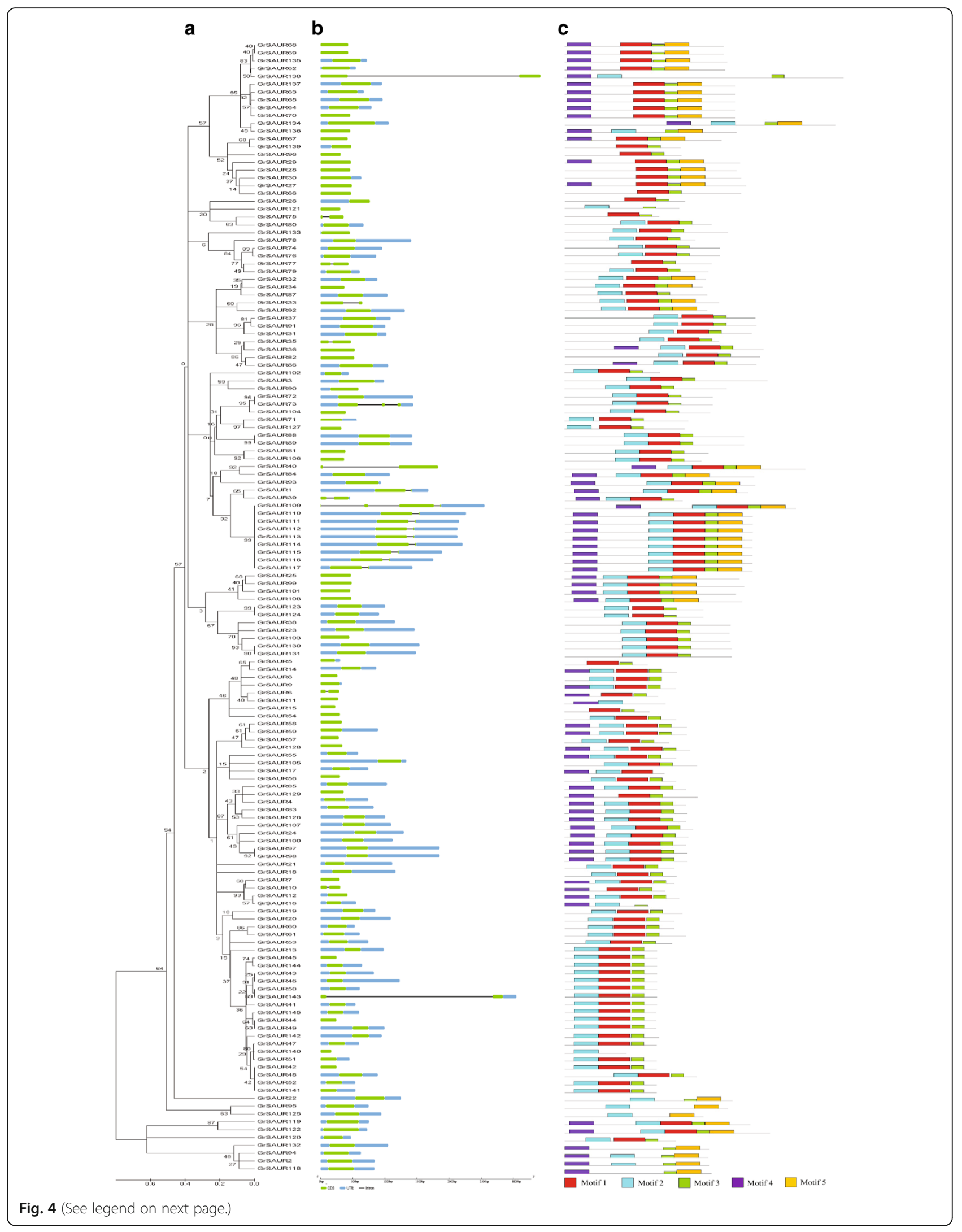


(See figure on previous page.)

Fig. 4 Phylogenetic relationships, gene structure and motif compositions of the Gossypium raimondii SAUR genes. a The phylogenetic tree was constructed using MEGA 6.0 with the Neighbour-Joining (NJ) method with 1000 bootstrap replicates. $\mathbf{b}$ Exon/intron structures of SAUR genes from G. raimondii. The introns, CDS and UTRs are represented by black lines, green and blue boxes respectively. The scale bar represents $0.5 \mathrm{~kb}$. $\mathbf{c}$ Protein motif. Each motif is represented in the colored box

compared with other genes. In the second group, 25 genes showed a progressive decrease from 0 DPA to 10 DPA, while 14 genes increased from 0 DPA to 10 DPA in the third group. The other 13 genes in group four showed an overall lower expression level. With the absolute value of $\log 2$-fold change $\geq 1$ which was also statistically significant as the standard to judge differently expressed genes (DEGs), we found that 6 GhSAUR genes were DEGs when compared between the two BILs at the same development stage, while 30 GhSAUR genes were found to be DEGs among the three different developmental stages (Fig. 6c). As shown in Fig. 6, common DEGs were detected among different comparisons.

In another RNA-seq transcriptome profiling, only 40 SAUR genes were found to be expressed in -3 or 0 DPA ovules of Xuzhou142 and its $f l$ mutant (Fig. 6b). Genes

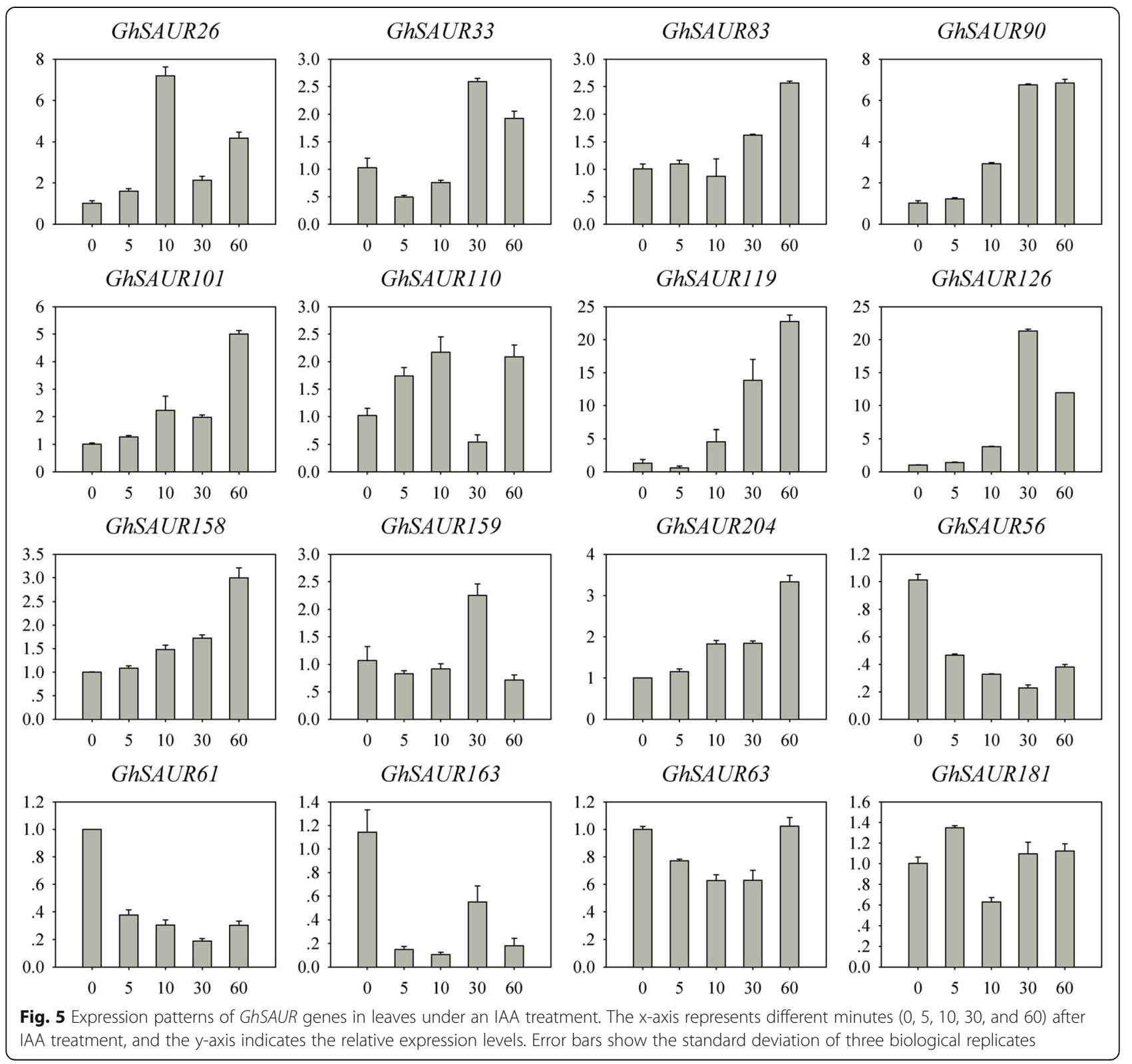


a
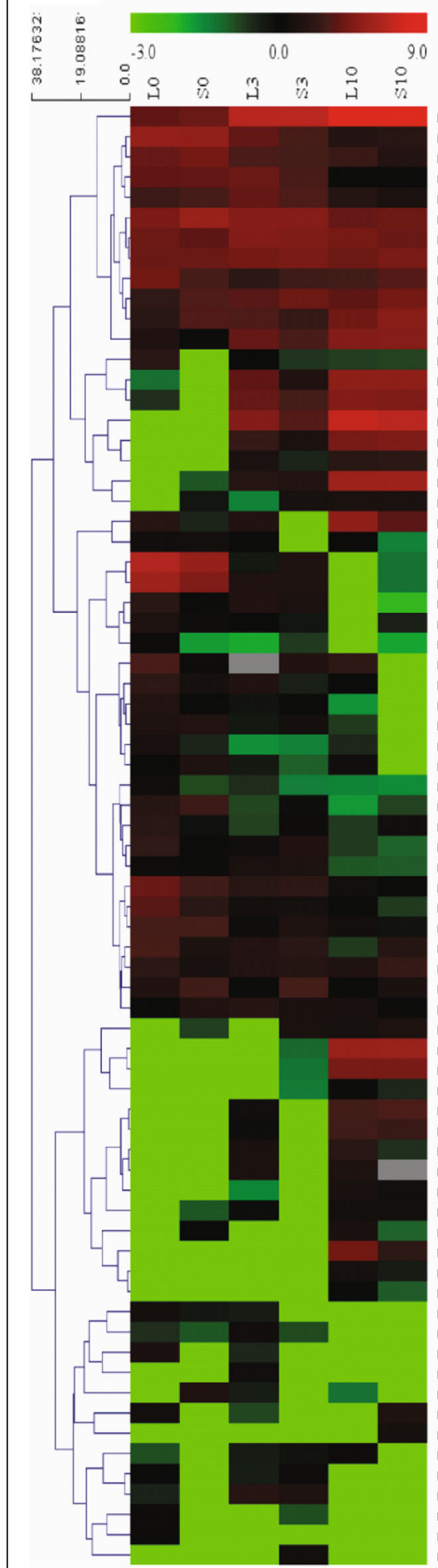

Fig. 6 (See legend on next page.)

\section{b \\ b}

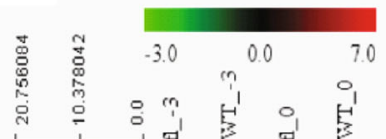

ChISAUR159
ChSAUR:5?

ChSAUR:134

ChSAURS?

ChSAUR?J

GhSAUR199

ChSAUR140

ChSAUR55

ChISAUR133

ChSAUR41

ChSAUR83

ChSAUR.25

ChSAUR:19

ChSAUR110

ChAUR

GhSAUR126

GhSAUR25

GhSAUR51

ChSAUR33

ChSAUR114

ChSAUR67
ChSAUR155

GhSAUR138

ChSAUR144

ChSAUR 167

GhSAUR148

ChSAUR194

ChSAUR6

GhSAURG

ChSAUR!

ChSAUR125

ChSAUR185

AUP186

ChSAUR7

ChSAUP71

ChSAUR 1

ChSAUR173

ChSAUR189

ChSAUR74

ChSAUR171

ChSAUR170

ChSAUR:158

ChSAUR196

ChSAUR55

ChSAUP1?0

GhSAUR2J1

ChSAUR116

GhSAUR11?

ChSAUR 31

GhSAUR?J7

ChSAUR113

ChSAUR13?

ChSAUR9J

ChSAUR149

ChSAUR:146

ChSAUR156

ChSAUR156

ChSAUR205

ChSAUR11

ChSAUR4]

ChSAUR79

ChSAUR181

ChSAUR109

ChSAUR54

ChSAUR62

ChSAUR179

ChSAUR3

ChSAUR127

ChSAURSS

I

C

III

IV
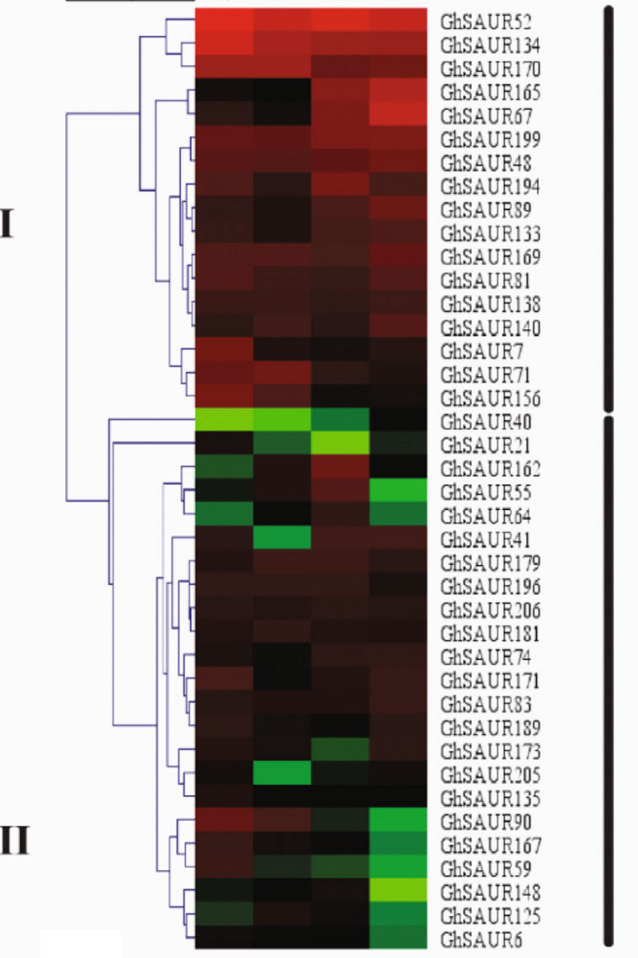

I

II
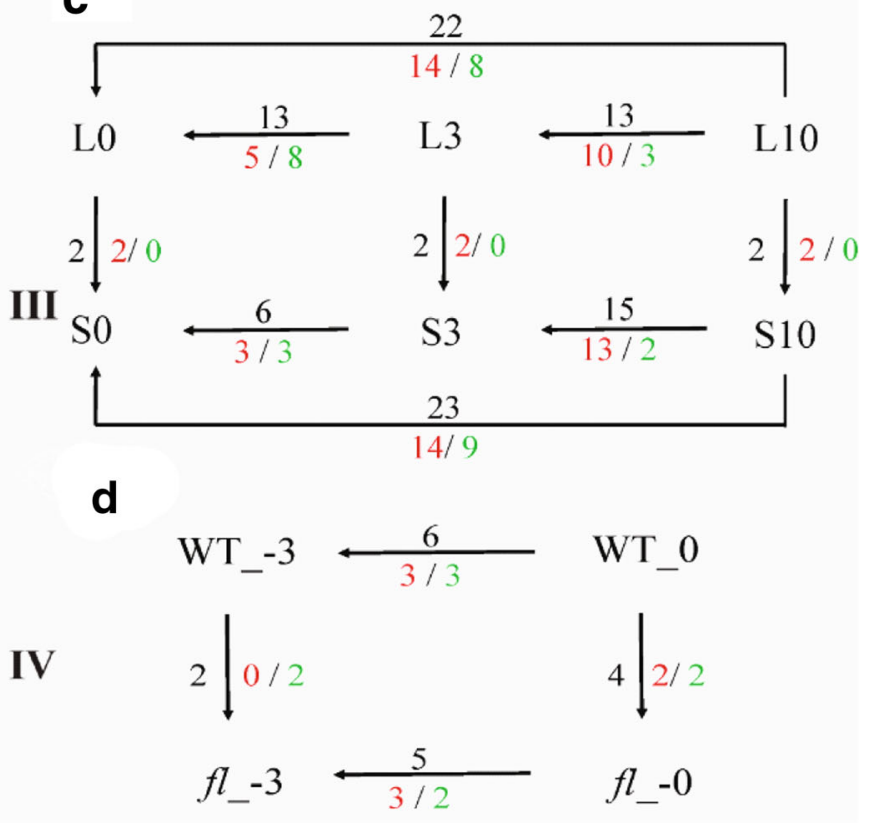
(See figure on previous page.)

Fig. 6 Expression profiles of GhSAUR genes based on RNA-seq data of two backcross inbred lines (BILs) and Xuzhou142 (WT) and Xuzhou142 fiberless and fuzzless (fl) mutant. a Transcript levels of 72 GhSAURs in three stages of two BILs. b Transcript levels of 40 GhSAURs in -3 and 0 DPA ovules of Xuzhou142 (WT) and its fl mutant. c The number of differentially expressed SAUR genes between different stages of BILs NMGA-062 with longer fibers (L) and NMGA-105 with shorter fibers (S). For example, L0 represents 0 DPA ovules of NMGA-062. d The number of differentially expressed SAUR genes between different stages of Xuzhou142 (WT) and Xuzhou142 fl mutant (ff). For example, WT_-3 represents -3 DPA ovules of Xuzhou142

showed a relatively higher level of expression in group one than in group two. Of 10 genes showing significant differential expressions, 2 and 4 DEGs were detected between Xuzhou 142 (WT) and its $f l$ mutant, at -3 and 0 DPA ovules, respectively, and 6 and 5 DEGs were detected between -3 and 0 DPA ovules in Xuzhou 142 (WT) and its $f l$ mutant, respectively. Interestingly, 3 of the 5-6 DEGs between -3 and 0 DPA ovules are in common in the two genotypes (Fig. 6d).
To further study the expression profiles based on RNAseq, quantitative RT-PCR (qRT-PCR) was conducted for 12 $S A U R$ genes in 5 organs and 8 fiber developmental stages of NMGA-062 (Fig. 7). The results showed that some SAUR genes exhibited diverse expression profiles, while others showed similar expression patterns. Specifically, four genes, GhSAUR62, GhSAUR158, GhSAUR126, and GhSAUR90, were exclusively highly expressed in stems or flowers. The expression of GhSAUR110, GhSAUR33,

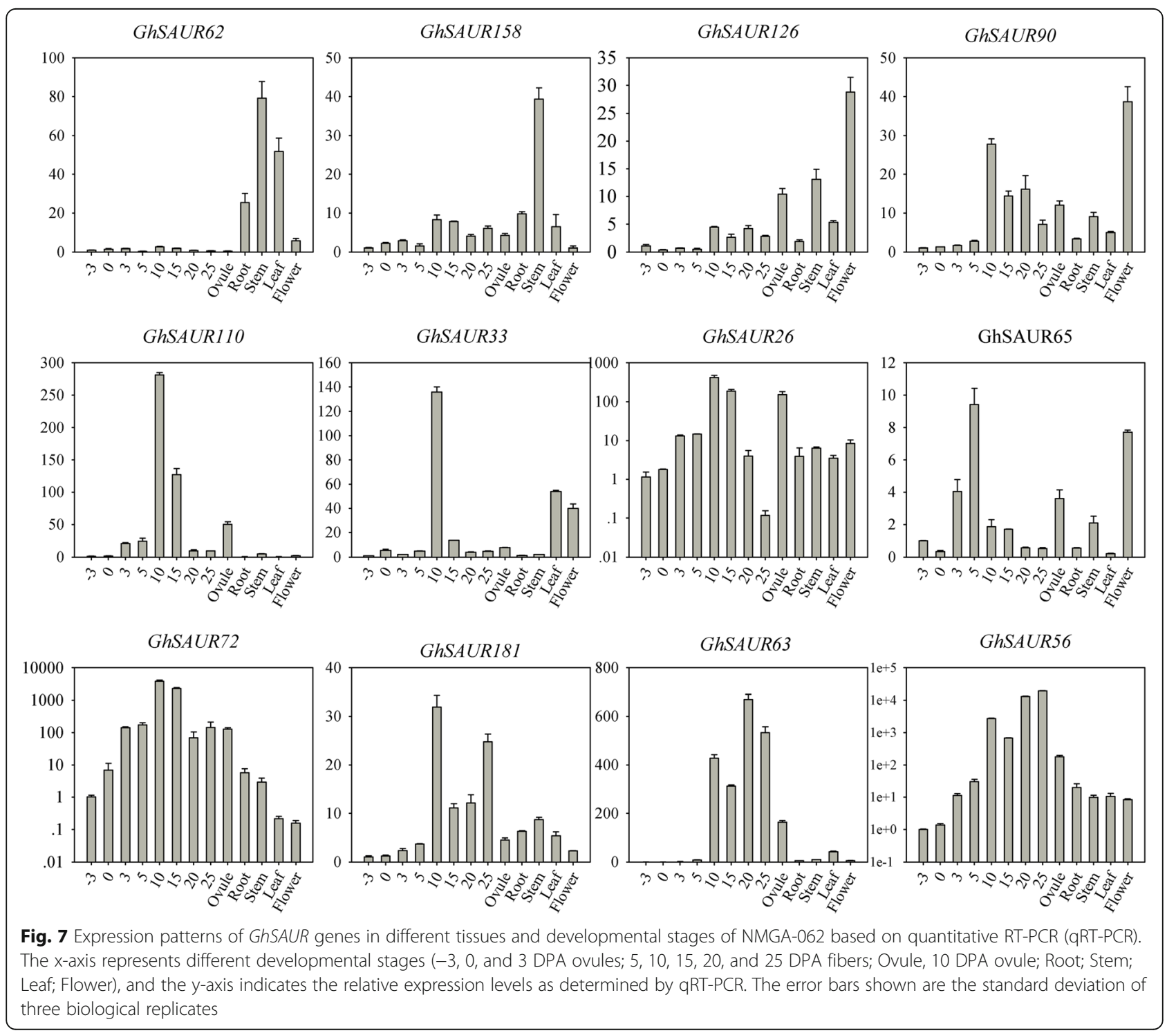


GhSAUR26, GhSAUR65, GhSAUR72, and GhSAUR181 increased between -3 and 20 DPA in that it peaked in fibers at 10 or $15 \mathrm{DPA}$, and then decreased at 20 DPA fibers. Another two genes, GhSAUR63 and GhSAUR56, were increased from -3 to 25 DPA with high expressions at 25 DPA fibers.

\section{Co-localization of SAURs with QTL for FL}

To better understand the potential function of SAUR genes related to fiber length (FL), we co-localized the SAUR genes with reported FL quantitative trait loci (QTL). As a result, 20 genes were mapped with the anchored FL QTL or FL QTL hotspots within a 25-cM region (Fig. 8). There was 1 gene (GhSAUR3) on chromosome AD1-A01, 1 gene (GhSAUR36) on AD1-A04, 1 gene (GhSAUR53) on AD1A07, and 2 genes (GhSAUR76 and GhSAUR77) on AD1A12 located within the FL QTL hotspots in the At subgenome. While 6 genes (i.e., GhSAUR128, GhSAUR129, GhSAUR130, GhSAUR131, GhSAUR132, and GhSAUR133) on chromosome AD1-D05, 5 genes (i.e., GhSAUR148, GhSAUR149, GhSAUR150, GhSAUR151, and GhSAUR152) on AD1-D08, 4 genes (i.e., GhSAUR171, GhSAUR172, GhSAUR173, and GhSAUR174) on AD1-D12 were located within the FL QTL hotspots in the Dt subgenome. Of these co-localized SAUR genes, only 3 genes were differentially expressed between the two BILs differing in fiber length. For example, GhSAUR149 was up-regulated in 10 DPA fibers of the BIL with longer fibers as compared with the BIL with shorter fibers, and its expression in the long fiber BIL was also higher in 10 DPA fibers than in 0 DPA ovules. However, GhSAUR148 in the long fiber BIL was down-regulated at 3 DPA ovules than at 0 DPA ovules; And GhSAUR173 in the long fiber BIL was also down-regulated at 3 ovules and 10 DPA fibers than 0 DPA ovules.

\section{Identification of single nucleotide polymorphisms (SNP)} in SAUR genes co-localized with FL

Sequence variations in the predicted SAUR genes among the sequenced G. raimondii (D5), G. arboreum (A2, Shixiya1), G. hirsutum (AD1, TM-1) and G. barbadense (AD2, 3-79 and Xinhai 21) were further analyzed (Additional file 12: Figure S5). 12 genes had 1-7 SNPs between AD1 and AD2, while other 8 genes were identical between AD1 and AD2. Among the SNP-containing SAUR genes, GhSAUR3 and GhSAUR77 from AD1 on the At subgenome have identical SNP sequences to the homologous genes in A2. Among 9 genes with homologous genes on D5, the SNP sites of GhSAUR174 in AD2 were identical to the homologous D5 genes, while the SNP sites of GhSAUR132, GhSAUR133, GhSAUR149 and GhSAUR171 in AD1 were identical to the homologous genes in D5.

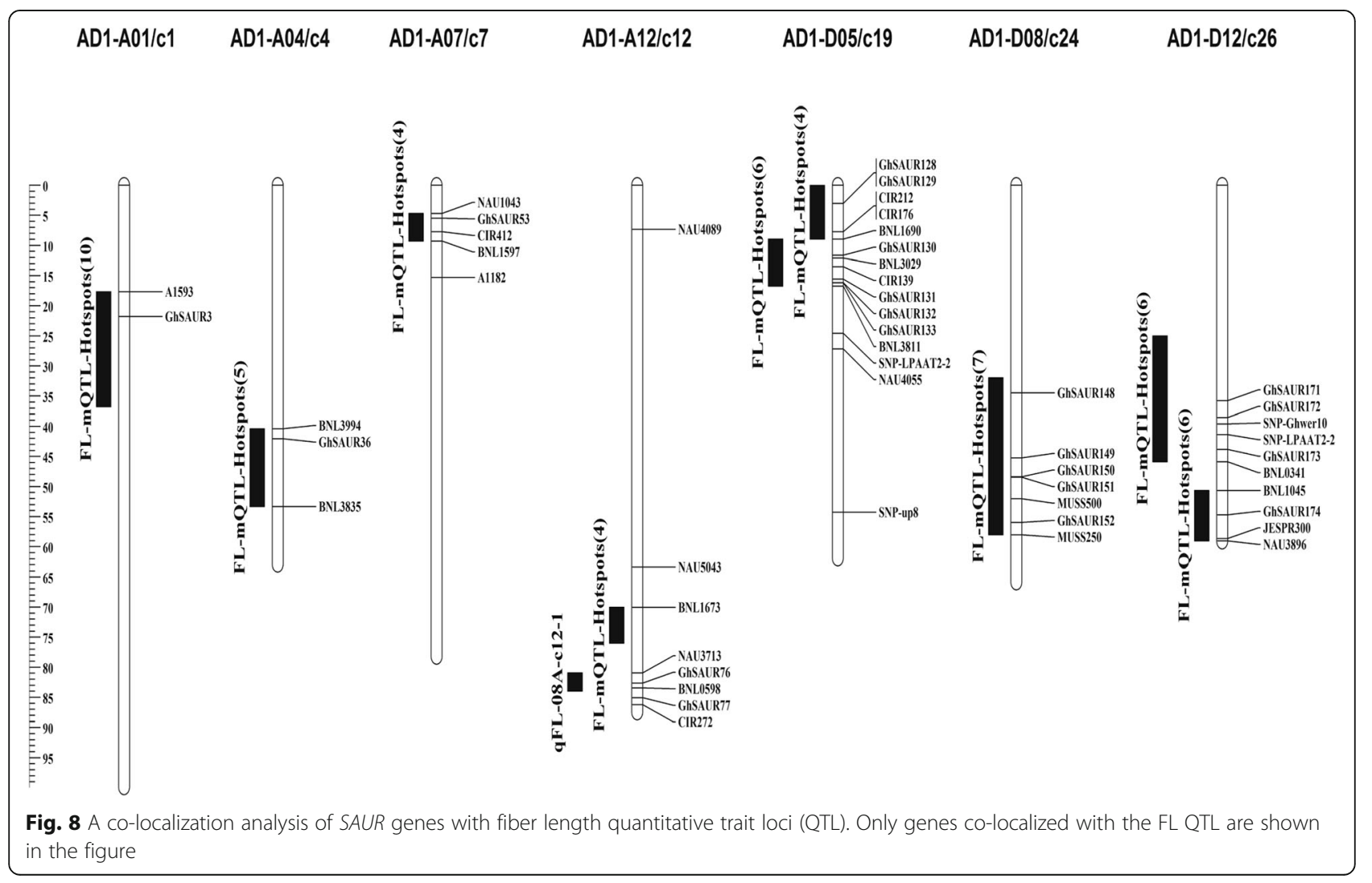


Interestingly, the SNP sites of homologous GhSAUR128, GhSAUR148, GhSAUR150 and GhSAUR152 genes in both AD1 and AD2 shared with D5 depending on SNP sites. 7 SNPs in GhSAUR53 from the At subgenome were detected between AD1 and AD2, but its sequence was different from both A2 and D5.

To understand if the co-localized SAURs are genetically associated with fiber elongation, a sequence comparison between NMGA-062 and NMGA-105 was performed, but no SNPs were identified. The results indicated that SAURs are not genetically related to fiber length, implying that the differences in fiber length between the two species are unlikely related to the natural sequence variations in the SAUR genes.

\section{Discussion}

\section{SAUR gene family in cotton}

Previous reports have suggested that the SAUR family regulates a series of cellular, physiological, and developmental processes in response to hormonal and environmental signals in higher plants [1]. However, the molecular network that links specific hormonal and environmental signals is still unknown. With the availability of genome sequences, a genome-wide identification and annotation of SAUR genes has been performed in Arabidopsis (72), rice (58), sorghum (71), tomato (99), potato (134), maize (79), citrus (70), mulberry (62), hemp (56), and ramie (71) [3, 6-11]. In this study, 145, 97, 214, and 176 SAUR genes in four sequenced cotton species, G. raimondii, G. arboreum, G. hirsutum, and G. barbadense, respectively, were identified in-silico and characterized. Compared with most of the SAUR gene family numbers in other reported species, more members were existent in cotton. It suggests that the SAUR family in cotton experienced an extensive expansion during its evolutionary history. It was reported that tandem and segmental duplication events contributed to the expansion of the SAUR family in Solanaceae species and maize $[8,9]$. In this current study, $55.7-87.4 \%$ of the SAUR gene family members in cotton were likely from tandem duplication and segmental duplication events. The duplication events occurred about 115-146 and 13-20 million year ago in G. arboreum and $G$. raimondii, respectively, which was followed by the formation of the G. hirsutum and G. barbadense from the hybridization of the two extant progenitors relatives and polyploidization event 1.5 million years ago $[23,26]$. Therefore, the SAUR gene number in the tetraploids G. hirsutum and G. barbadense is likely to depend on the number of G. arboreum and G. raimondii. Segmental duplications can further contribute to the expansion of the SAUR family. The chromosomal distribution of SAUR gene family also showed that genes in this family were not randomly distributed on the genome, but in tandem arrays of extremely related paralogous genes, as reported in other species $[3,6]$. Thus, tandem duplication and segmental duplication events also contributed to expansion of SAUR family in cotton, as other reported gene families [30, 32].

\section{Genomic structure of SAUR family genes}

The majority of SAUR gene family lacks introns. Only one SAUR gene in Arabidopsis has an intron, while none of the OsSAURs in rice harbors any intron. Among other species, 6 out of 58 SAUR genes in maize, 3 out of 99 SAUR genes in tomato, 9 out of SAUR genes in potato, 10 out of 70 SAUR genes in citrus contained introns based on the sequenced genomes [3, 6, 8-10]. Similar phenomenon in cotton was found in this study in that about $9.3 \%$ of the SAUR genes in cotton carried introns. As the occurrence of alternative splice in intronless genes is usually low, the function of certain SAUR family genes is likely stable.

The motif analysis of the sequenced cotton species showed the existence of the conserved 60 amino acid domain with three motifs specific to the SAUR proteins, similar to SAURs in tomato, potato, tobacco, rice, sorghum and Arabidopsis [8]. Although SAUR proteins have variable $\mathrm{N}$ - and $\mathrm{C}$-terminal extensions, the relatively short sequence lengths render a high level of similarity between SAUR genes.

\section{Expression profiles and putative functions of GhSAURs}

As an important type of auxin responsive genes, SAURs participate in the auxin signal pathway. The cis-element analysis in putative promoter regions of the GhSAURs showed that most genes possess at least one type of auxin-responsive cis-elements. We analyzed the expression levels of randomly chosen 16 GhSAUR genes under IAA treatment. The expression levels of 11 analyzed genes were up-regulated, while 3 were down-regulated after the treatment. This confirmed previous reports that SAUR genes were upregulated or repressed to some extent following an auxin treatment $[1,11]$.

Auxin plays an important role in fiber development, as shown by a previous report that the lint yield and fiber fineness were improved with the overexpression of the IAA biosynthetic gene iaaM, driven by the promoter from the petunia MADS box gene Floral Binding protein 7 [22]. The application of auxin increased fiber units and promoted fiber initiation in in-vitro cultured cotton ovules [20]. In this study, the expression profiles of SAUR genes in two BILs (differing in fiber length) were analyzed in fiber initiation and elongation stage. We found many SAUR genes in group II and III showed differential expressions from 0 DPA to 10 DPA (Fig. 6). We suggest that these genes may be regulated during fiber initiation and elongation. We also showed that several 
genes were differentially expressed at the fiber initiation stage between Xuzhou142 and its $f l$ mutant.

We also investigated the gene expression patterns of 12 SAUR genes in various tissues. As Fig. 7 showed, apart from four genes that had a relatively high expression level in stems and flowers, the other tested genes had high levels in different fiber development stages ( -3 to $25 \mathrm{DPA}$ ). The alignment of these genes with Arabidopsis and rice SAUR genes showed that GhSAUR33 had a high similarity with AtSAUR61-AtSAUR68 subfamily and OsSAUR54. In Arabidopsis, transgenic plants expressing SAUR63:GFP or SAUR63:GUS fusions had long hypocotyls, petals and stamen filaments, while overexpressed artificial microRNAs targeting SAUR63 subfamily led to reduced hypocotyls and stamen filament elongation. The results indicated that these AtSAUR genes regulated cell expansion to change the hypocotyl growth [15]. OsSAUR54 was preferentially expressed in rice stigma, and may promote pollen tube growth [47]. Cotton fibers are single-celled trichomes, and grow via a similar mode to pollen tubes in that the fiber cell elongation depends on cell expansion [48]. Our qRT-PCR result and the function of orthologous genes of GhSAUR33 indicated that it may have a similar function in cotton fiber elongation.

In cotton, the auxin signaling pathway was associated with the dedifferentiation and redifferentiation during somatic embryogenesis in a transcript profiling analysis on SAUR genes [49]. In another study, SAUR genes were found down-regulated in a dwarf cotton genotype when compared with the wild type, indicating their involvement in the growth of plant height [50]. In Arabidopsis, SAUR genes were reported to regulate plant growth and development via regulating cell expansion [12-14], shade avoidance responses [51], tropic growth [52], root growth [18], auxin transport [15], and leaf growth and senescence [16]. As a plant specific gene family, SAURs in cotton are also likely to have diverse functions. However, no other researches about SAUR genes were reported in cotton except for the above two reports. Therefore, our study provides an important piece of information that will facilitate our understanding of specific functions of SAURs in cotton growth and development.

\section{Conclusions}

This study provides a comprehensive analysis of SAUR gene family in sequenced genomes of four cotton species for the first time. The phylogenetic analysis of SAURs classified the SAUR genes into 10 groups. A chromosomal location and gene duplication analysis revealed that duplication events have contributed to the expansion of the SAUR gene family in cotton. Most studied GhSAUR genes showed differential expressions in leaves in response to auxin applications. A further expression analysis using RNA-seq transcriptome and qRT-PCR showed various expression patterns of SAUR genes in early developmental ovules and fibers. Although 20 SAURs are co-localized with fiber length quantitative trait loci (QTL), no sequence variations were identified between two interspecific backcross inbred lines (BILs) with different fiber length, suggesting an unlikely genetic involvement of these SAURs genes in fiber elongation.

\section{Additional files}

Additional file 1: Table S1. Primers used for qRT-PCR analysis. (XLSX $9 \mathrm{~kb}$ ) Additional file 2: Table S2. SAUR gene family in Gossypium arboreum. (XLS $47 \mathrm{~kb})$

Additional file 3: Table S3. SAUR gene family in Gossypium hirsutum. (XLSX $28 \mathrm{~kb}$ )

Additional file 4: Table S4. SAUR gene family in Gossypium barbadense. (XLSX $25 \mathrm{~kb}$ )

Additional file 5: Table S5. The number of SAUR genes from 12 species classified into groups I - X. (XLSX $9 \mathrm{~kb}$ )

Additional file 6: Table S6. The paralogous pairs of SAUR genes in Gossypium raimondii, G. arboreum, G. hirsutum, and G. barbadense. (XLSX $12 \mathrm{~kb}$ )

Additional file 7: Figure S1. Phylogenetic relationships, gene structure and motif compositions of the Gossypium arboreum SAUR genes. (A) The phylogenetic tree was constructed using MEGA 6.0 with the Neighbour-Joining (NJ) method with 1000 bootstrap replicates. (B) Exon/intron structures of SAUR genes from G. arboreum. The introns, CDS are represented by black lines, green and blue boxes respectively. The scale bar represents $0.5 \mathrm{~kb}$. (C) Protein motif. Each motif is represented in the colored box. (PDF 2081 kb)

Additional file 8: Figure S2. Phylogenetic relationships, gene structure and motif compositions of the Gossypium hirsutum SAUR genes. (A) The phylogenetic tree was constructed using MEGA 6.0 with the NeighbourJoining (NJ) method with 1000 bootstrap replicates. (B) Exon/intron structures of SAUR genes from $G$. hirsutum. The introns, CDS are represented by black lines, green and blue boxes respectively. The scale bar represents $0.5 \mathrm{~kb}$. (C) Protein motif. Each motif is represented in the colored box. (PDF 509 kb)

Additional file 9: Figure S3. Phylogenetic relationships, gene structure and motif compositions of the Gossypium barbadense SAUR genes. (A) The phylogenetic tree was constructed using MEGA 6.0 with the Neighbour-Joining (NJ) method with 1000 bootstrap replicates. (B) Exon/ intron structures of SAUR genes from G. hirsutum. The introns, exons and UTRs are represented by black lines, green and blue boxes respectively. The scale bar represents $0.5 \mathrm{~kb}$. (C) Protein motif. Each motif is represented in the colored box. (PDF $677 \mathrm{~kb}$ )

Additional file 10: Figure S4. A multiple sequence alignment of histidine-rich SAUR proteins in Gossypium raimondii, G. arboreum, G. hirsutum, and G. barbadense. The histidine-rich regions are indicated by lines above the sequences. (PDF $769 \mathrm{~kb}$ )

Additional file 11: Table S7. Cis-elements in the promoters of GhSAUR genes. (XLSX $15 \mathrm{~kb}$ )

Additional file 12: Figure S5. Sequence alignment of the predicted SAUR genes between four sequenced Gossypium species. The red boxes indicate sequence variations of G. hirsutum (TM-1) and G. barbadense (3-79 and Xinhai 21). Gh1 indicate TM-1 sequenced by Zhang et al. (2015). Gb1 and Gb2 indicate 3-79 and Xinhai21 sequenced by Yuan et al. (2015) and Liu et al. (2015), respectively. Gr and Ga indicate two diploid genomes sequenced by Paterson et al. (2013) and Li et al. (2014), respectively. (PDF $335 \mathrm{~kb}$ )

\section{Abbreviations}

BILs: Backcross inbred lines; DEGs: Differentially expressed genes; DPA: Days post anthesis; FL: Fiber length; FPKM: Fragments per kilobase of transcript per million fragments; QTL: Quantitative trait loci; SAUR: Small auxin-up RNA; SNP: Single nucleotide polymorphism 


\section{Acknowledgements}

The authors wish to thank New Mexico Agricultural Experiment Station, New Mexico, USA.

\section{Funding}

This research was support by the National Natural Science Foundation of China (grant No. 31621005) and the National Key Research and Development Program of China (grant No. 2016YFD0101400).

\section{Availability of data and materials}

The sequence read data from RNA-seq analysis for the 2 BILs are available in the Sequence Read Archive (SRA) (accession number SRP039385 and SRP038911). While the sequence data for Xuzhou 142 and Xuzhou $142 \mathrm{fl}$ can be accessed through accession number SRP056184 The data sets supporting the results of this study are included in the manuscript and its additional files.

\section{Authors' contributions}

JWY, SXY and JFZ conceived and designed the experiments. MW, WFP and $X L L$ cultivated the cotton plants in the field and collected the fiber and ovule samples. HHZ and XSZ collected the leaves samples in the culture room. GYL and YHG performed the GRT-PCR experiments. XHL performed the data analysis and wrote the manuscript. JFZ revised the manuscript. All authors reviewed and approved the manuscript.

\section{Ethics approval and consent to participate}

Not applicable.

\section{Consent for publication}

Not applicable.

\section{Competing interests}

The authors declare that they have no competing interests.

\section{Publisher's Note}

Springer Nature remains neutral with regard to jurisdictional claims in published maps and institutional affiliations.

\section{Author details}

'State Key Laboratory of Cotton Biology, Institute of Cotton Research of CAAS, Anyang 455000, China. ${ }^{2}$ College of Agronomy, Northwest A\&F University, Yangling 712100, China. ${ }^{3}$ Department of Plant and Environmental Sciences, New Mexico State University, Las Cruces 88003, USA.

\section{Received: 1 February 2017 Accepted: 17 October 2017}

\section{Published online: 23 October 2017}

\section{References}

1. Ren $H$, Gray WM. SAUR proteins as effectors of hormonal and environmental signals in plant growth. Mol Plant. 2015;8(8):1153-64.

2. Abel S, Oeller PW, Theologis A. Early auxin-induced genes encode shortlived nuclear proteins. Proc Natl Acad Sci U S A. 1994;91(1):326-30.

3. Hagen G, Guilfoyle T. Auxin-responsive gene expression: genes, promoters and regulatory factors. Plant Mol Biol. 2002;49(3-4):373-85.

4. Newman TC, Ohme-Takagi M, Taylor CB, Green PJ. DST sequences, highly conserved among plant SAUR genes, target reporter transcripts for rapid decay in tobacco. Plant Cell. 1993;5(6):701-14.

5. McClure BA, Guilfoyle T. Characterization of a class of small auxin-inducible soybean polyadenylated RNAs. Plant Mol Biol. 1987;9(6):611-23.

6. Jain M, Tyagi AK, Khurana JP. Genome-wide analysis, evolutionary expansion, and expression of early auxin-responsive SAUR gene family in rice (Oryza sativa). Genomics. 2006;88(3):360-71.

7. Wang S, Bai Y, Shen C, Wu Y, Zhang S, Jiang D, Guilfoyle TJ, Chen M, Qi Y. Auxin-related gene families in abiotic stress response in Sorghum Bicolor. Funct Integr Genomics. 2010;10(4):533-46.

8. Wu J, Liu S, He Y, Guan X, Zhu X, Cheng L, Wang J, Lu G. Genome-wide analysis of SAUR gene family in Solanaceae species. Gene. 2012;509(1):38-50.

9. Chen Y, Hao X, Cao J. Small auxin upregulated RNA (SAUR) gene family in maize: identification, evolution, and its phylogenetic comparison with Arabidopsis, rice, and sorghum. J Integr Plant Biol. 2014;56(2):133-50.
10. Xie R, Dong C, Ma Y, Deng L, He S, Yi S, Lv Q, Zheng Y. Comprehensive analysis of SAUR gene family in citrus and its transcriptional correlation with fruitlet drop from abscission zone A. Funct Integr Genomics. 2015;15(6):729-40.

11. Huang X, Bao Y, Wang BO, Liu L, Chen J, Dai L, Baloch SU, Peng D. Identification of small auxin-up RNA (SAUR) genes in Urticales plants: mulberry (Morus Notabilis), hemp (Cannabis sativa) and ramie (Boehmeria nivea). J Genet. 2016;95(1):119-29.

12. Spartz AK, Lee SH, Wenger JP, Gonzalez N, Itoh H, Inze D, Peer WA, Murphy AS, Overvoorde PJ, Gray WM. The SAUR19 subfamily of SMALL AUXIN UP RNA genes promote cell expansion. Plant J. 2012;70(6):978-90.

13. Spartz AK, Ren H, Park MY, Grandt KN, Lee SH, Murphy AS, Sussman MR, Overvoorde PJ, Gray WM. SAUR inhibition of PP2C-D phosphatases activates plasma membrane $\mathrm{H}+-$ ATPases to promote cell expansion in Arabidopsis. Plant Cell. 2014;26(5):2129-42.

14. Spartz AK, Lor VS, Ren H, Olszewski NE, Miller ND, Wu G, Spalding EP, Gray WM: Constitutive expression of Arabidopsis small Auxin up RNA19 (SAUR19) in tomato confers auxin-independent hypocotyl elongation. Plant Physiol. 2017:173:1453-62.

15. Chae K, Isaacs CG, Reeves PH, Maloney GS, Muday GK, Nagpal P, Reed JW. Arabidopsis SMALL AUXIN UP RNA63 promotes hypocotyl and stamen filament elongation. Plant J. 2012;71(4):684-97.

16. Hou K, Wu W, Gan SS. SAUR36, a small auxin up RNA gene, is involved in the promotion of leaf senescence in Arabidopsis. Plant Physiol. 2013;161(2):1002-9.

17. Kant S, Bi YM, Zhu T, Rothstein SJ. SAUR39, a small auxin-up RNA gene, acts as a negative regulator of auxin synthesis and transport in rice. Plant Physiol. 2009;151(2):691-701.

18. Li ZG, Chen HW, Li QT, Tao JJ, Bian XH, Ma B, Zhang WK, Chen SY, Zhang JS. Three SAUR proteins SAUR76, SAUR77 and SAUR78 promote plant growth in Arabidopsis. Sci Rep. 2015;5:12477.

19. Sun N, Wang J, Gao Z, Dong J, He H, Terzaghi W, Wei N, Deng XW, Chen $\mathrm{H}$. Arabidopsis SAURs are critical for differential light regulation of the development of various organs. Proc Natl Acad Sci U S A. 2016; 113(21):6071-6.

20. Beasley CA. Hormonal regulation of growth in unfertilized cotton ovules. Science. 1973:179(4077):1003-5.

21. Guinn G, Brummett DL. Changes in abscisic acid and indoleacetic acid before and after anthesis relative to changes in abscission rates of cotton fruiting forms. Plant Physiol. 1988;87(3):629-31.

22. Zhang $M$, Zheng $X$, Song $S$, Zeng $Q$, Hou L, Li D, Zhao J, Wei Y, Li X, Luo M, et al. Spatiotemporal manipulation of auxin biosynthesis in cotton ovule epidermal cells enhances fiber yield and quality. Nat Biotechnol. 2011;29(5):453-8.

23. Li F, Fan G, Lu C, Xiao G, Zou C, Kohel RJ, Ma Z, Shang H, Ma X, Wu J, et al. Genome sequence of cultivated Upland cotton (Gossypium hirsutum TM-1) provides insights into genome evolution. Nat Biotechnol. 2015;33(5):524-30.

24. Zhang T, Hu Y, Jiang W, Fang L, Guan X, Chen J, Zhang J, Saski CA, Scheffler BE, Stelly DM, et al. Sequencing of allotetraploid cotton (Gossypium hirsutum L. acc. TM-1) provides a resource for fiber improvement. Nat Biotechnol. 2015;33(5):531-7.

25. Yuan D, Tang Z, Wang M, Gao W, Tu L, Jin X, Chen L, He Y, Zhang L, Zhu L, et al. The genome sequence of Sea-Island cotton (Gossypium barbadense) provides insights into the allopolyploidization and development of superior spinnable fibres. Sci Rep. 2015;5:17662.

26. Liu X, Zhao B, Zheng HJ, Hu Y, Lu G, Yang CQ, Chen JD, Chen JJ, Chen DY, Zhang L, et al. Gossypium barbadense genome sequence provides insight into the evolution of extra-long staple fiber and specialized metabolites. Sci Rep. 2015;5:14139.

27. Paterson AH, Wendel JF, Gundlach $H$, Guo H, Jenkins J, Jin D, Llewellyn D, Showmaker KC, Shu S, Udall J, et al. Repeated polyploidization of Gossypium genomes and the evolution of spinnable cotton fibres. Nature. 2012;492(7429):423-7.

28. Wang K, Wang Z, Li F, Ye W, Wang J, Song G, Yue Z, Cong L, Shang H, Zhu S, et al. The draft genome of a diploid cotton Gossypium raimondii. Nat Genet. 2012;44(10):1098-103.

29. Li F, Fan G, Wang K, Sun F, Yuan Y, Song G, Li Q, Ma Z, Lu C, Zou C, et al. Genome sequence of the cultivated cotton Gossypium arboreum. Nat Genet. 2014:46(6):567-72

30. Wang X, Ma Q, Dou L, Liu Z, Peng R, Yu S. Genome-wide characterization and comparative analysis of the MLO gene family in cotton. Plant Physiol Biochem. 2016;103:106-19. 
31. Zhang X, Wang L, Xu X, Cai C, Guo W. Genome-wide identification of mitogen-activated protein kinase gene family in Gossypium raimondii and the function of their corresponding orthologs in tetraploid cultivated cotton. BMC Plant Biol. 2014;14:345.

32. Niu E, Cai C, Zheng Y, Shang X, Fang L, Guo W. Genome-wide analysis of CrRLK1L gene family in Gossypium and identification of candidate CrRLK1L genes related to fiber development. Mol Genet Genomics. 2016;291(3):1137-54.

33. Yu J, Jung S, Cheng CH, Ficklin SP, Lee T, Zheng P, Jones D, Percy RG, Main D. CottonGen: a genomics, genetics and breeding database for cotton research. Nucleic Acids Res. 2014;42(Database issue):D1229-36. https://www. cottongen.org/

34. Finn RD, Bateman A, Clements J, Coggill P, Eberhardt RY, Eddy SR, Heger A, Hetherington K, Holm L, Mistry J, et al. Pfam: the protein families database. Nucleic Acids Res. 2014;42(Database issue):D222-30. http://pfam.xfam.org/

35. Artimo $P$, Jonnalagedda M, Arnold K, Baratin D, Csardi G, de Castro E, Duvaud S, Flegel V, Fortier A, Gasteiger E et al: ExPASy: SIB bioinformatics resource portal. Nucleic Acids Res 2012, 40(Web Server issue):W597-W603. http://web.expasy.org/protparam/

36. Yu CS, Lin CJ, Hwang JK. Predicting subcellular localization of proteins for gram-negative bacteria by support vector machines based on n-peptide compositions. Protein Sci. 2004;13(5):1402-6. http://cello.life.nctu.edu.tw/

37. Tamura K, Stecher G, Peterson D, Filipski A, Kumar S. MEGA6: Molecular evolutionary genetics analysis version 6.0. Mol Biol Evol. 2013;30(12):2725-9. http://www.megasoftware.net/

38. Voorrips RE. MapChart: software for the graphical presentation of linkage maps and QTLs. J Hered. 2002;93(1):77-8.

39. Hu B, Jin JP, Guo AY, Zhang H, Luo JC, Gao G. GSDS 2.0: an upgraded gene feature visualization server. Bioinformatics. 2015;31(8):1296-97. http://gsds. cbi.pku.edu.cn/.

40. Higo K, Ugawa Y, Iwamoto M, Korenaga T. Plant cis-acting regulatory DNA elements (PLACE) database. Nucleic Acids Research. 1999;27(1):297-300. https://sogo.dna.affrc.go.jp/cgi-bin/sogo.cgi?lang=en\&pj=640\&action= page\&page=newplace.

41. Yu J, Zhang K, Li S, Yu S, Zhai H, Wu M, Li X, Fan S, Song M, Yang D, et al. Mapping quantitative trait loci for lint yield and fiber quality across environments in a Gossypium hirsutum x Gossypium barbadense backcross inbred line population. Theor Appl Genet. 2013;126(1):275-87.

42. Li X, Wu M, Liu G, Pei W, Zhai H, Yu J, Zhang J, Yu S. Identification of candidate genes for fiber length quantitative trait loci through RNA-Seq and linkage and physical mapping in cotton. BMC Genomics. 2017;18(1):427.

43 de Hoon MJL, Imoto S, Nolan J, Miyano S: Open source clustering software. Bioinformatics, 2004, 20(9):1453-54. http://bonsai.hgc.jp/ mdehoon/ software/cluster/.

44. Guan X, Pang M, Nah G, Shi X, Ye W, Stelly DM, Chen ZJ. miR828 and miR858 regulate homoeologous MYB2 gene functions in Arabidopsis trichome and cotton fibre development. Nat Commun. 2014;5:3050.

45. Wang N, Ma J, Pei W, Wu M, Li H, Li X, Yu S, Zhang J, Yu J. A genome-wide analysis of the lysophosphatidate acyltransferase (LPAAT) gene family in cotton: organization, expression, sequence variation, and association with seed oil content and fiber quality. BMC Genomics. 2017;18(1):218.

46. Said Jl, Song M, Wang H, Lin Z, Zhang X, Fang DD, Zhang J. A comparative meta-analysis of QTL between intraspecific Gossypium Hirsutum and interspecific Gossypium hirsutum x G. barbadense populations. Mol Genet Genomics. 2015;290(3):1003-25.

47. Li M, Xu W, Yang W, Kong Z, Xue Y: Genome-wide gene expression profiling reveals conserved and novel molecular functions of the stigma in rice. Plant Physiol. 2007;144(4):1797-812.

48. Qin YM, Zhu YX. How cotton fibers elongate: a tale of linear cell-growth mode. Curr Opin Plant Biol. 2011;14(1):106-11.

49. Yang $X$, Zhang $X$, Yuan $D$, Jin $F$, Zhang $Y, X u$ J. Transcript profiling reveals complex auxin signalling pathway and transcription regulation involved in dedifferentiation and redifferentiation during somatic embryogenesis in cotton. BMC Plant Biol. 2012;12:110.

50. An W, Gong W, He S, Pan Z, Sun J, Du X. MicroRNA and mRNA expression profiling analysis revealed the regulation of plant height in Gossypium hirsutum. BMC Genomics. 2015;16:886.
51. de Wit M, Lorrain S, Fankhauser C: Auxin-mediated plant architectural changes in response to shade and high temperature. Physiol Plant 2014, 151(1):13-24.

52. Qiu T, Chen Y, Li M, Kong Y, Zhu Y, Han N, Bian H, Zhu M, Wang J. The tissue-specific and developmentally regulated expression patterns of the SAUR41 subfamily of small auxin up RNA genes: potential implications. Plant Signal Behav. 2013;8(8):e25283.

\section{Submit your next manuscript to BioMed Central and we will help you at every step:}

- We accept pre-submission inquiries

- Our selector tool helps you to find the most relevant journal

- We provide round the clock customer support

- Convenient online submission

- Thorough peer review

- Inclusion in PubMed and all major indexing services

- Maximum visibility for your research

Submit your manuscript at www.biomedcentral.com/submit
Biomed Central 